\title{
Molecular Structures and Dynamics of the Stepwise Activation Mechanism of a Matrix Metalloproteinase Zymogen: Challenging the Cysteine Switch Dogma
}

Gabriel Rosenblum, Samy Meroueh, Marta Toth, Jed F. Fisher, Rafael Fridman, Shahriar Mobashery, Irit Sagi

\section{N-terminal sequencing}

Pro-MMP-9 was incubated with its endogenous activator tissue kallikrein for 2 and 10min at a protein:activator ratio of 1:6. The mixture was analyzed by SDS-PAGE (Figure S1) and the excised bands were subjected to N-terminal sequencing. Sequencing of the intermediary band revealed that this MMP-9 form is a result of cleavage of LeuSer at positions $78-79$, located at the end of the second helix of the pro-peptide, giving rise to $85 \mathrm{kDa}$ species. Longer incubation periods lead to the formation of another band ascribed to the active species (see text). Sequence analysis revealed that the second cleavage site is located at Phe-Gln at positions 107-108, centered in a long loop connecting the third loop of the pro-peptide and the first strand of the active site. The second proteolytic cleavage is located downstream to the zinc-coordinated-Cys-99 and it yields the $82 \mathrm{kDa}$ active form.

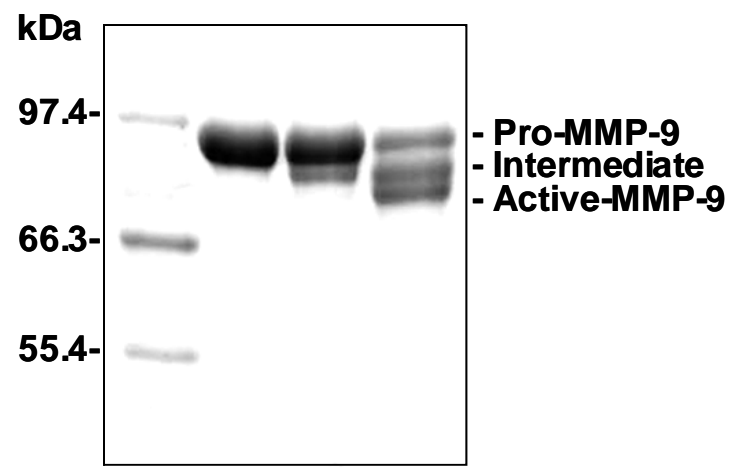

Figure S1. Limited activation of pro-MMP-9 by tissue-kallikrein. Pro-MMP-9 (lane 1) was incubated for $2 \mathrm{~min}$ (lane 2) and 10min (lane 3) with tissue kallikrein and the reaction mixture was subjected to SDSPAGE. Protein bands corresponding to the intermediate and fully activated MMP-9 were analyzed by Nterminal sequencing (see Materials and Methods). 


\section{Protein-Protein Docking}

Protein-protein docking involves both geometric and electrostatic consideration in order to generate solutions (The 3D-Dock suite was used ${ }^{2-4}$ ). These solutions were further filtered according to the employed biochemical and structural knowledge where the catalytic triad of tissue-kallikrein should hydrolyze the first cleavage site of the propeptide. Specifically, the first step of the proteolytic reaction is the interaction of Ser-195 within the catalytic triad of tissue-kallikrein with Leu-78 carbonyl within the cleavage site located at the pro-peptide of MMP-9. All solution complexes indicate that a separation of 12 to $15 \AA$ exist between the interacting residues. Therefore, the question to be asked relate to the occurrence of a cleavage when: (1) the cleavage site of pro-MMP-9 is separated from the catalytic triad. (2) The cleavage site of pro-MMP-9 is partially buried. We proposed that the pro-peptide needs to undergo a conformational change that exposes the cleavage site and allow it to reach the catalytic triad of tissue-kallikrein. The chronology of events described here support this assumption by showing that the $\mathrm{Zn}$ $\mathrm{S}($ Cys-99) dissociation is completed after $300 \mathrm{~ms}$, whereas the dissociation of the first segment of the pro-peptide exhibit slower kinetics with tens-of-seconds time frame. This might suggest that the $\mathrm{Zn}-\mathrm{S}(\mathrm{Cys}-99)$ dissociation serves as a prerequisite for such a conformational change that finally allow the proteolytic cleavage and predisposal of the first pro-peptide segment.

The docking results are represented in Figure 4 in the text. Additional views of these results are shown in Figure S2. 

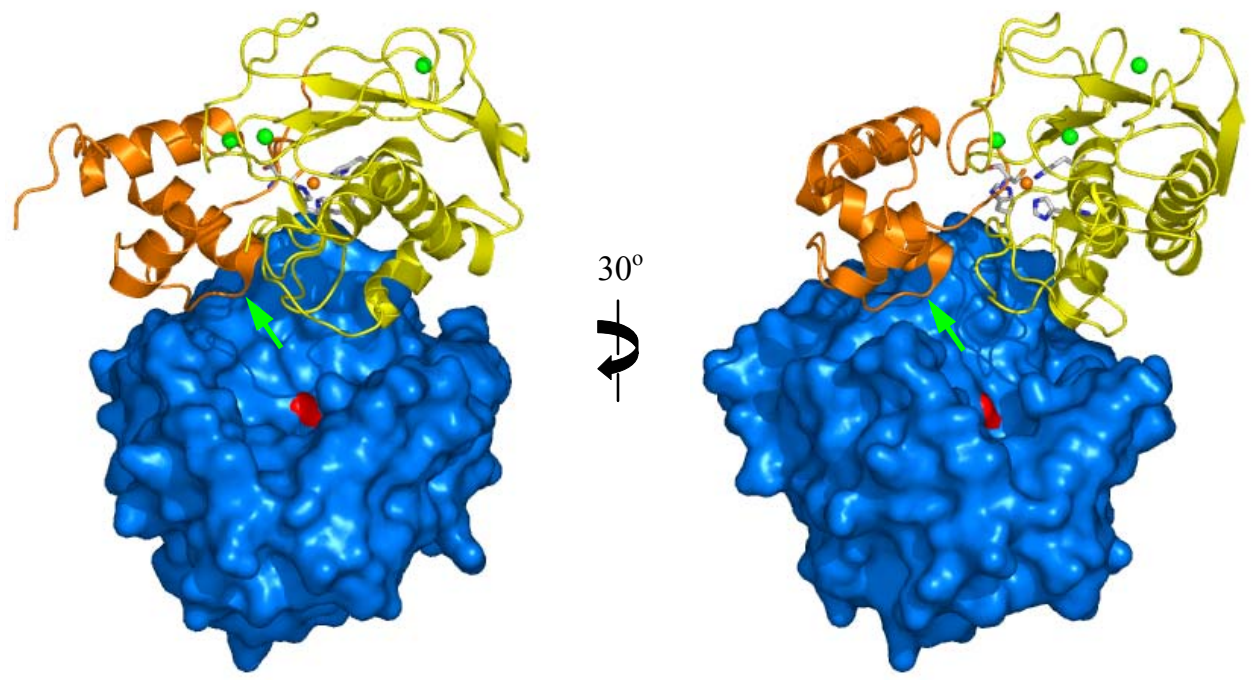

Figure S2. Docking result of tissue kallikrein to pro-MMP-9. The two views of the docking result are rotated vertically by $30^{\circ}$. Tissue kallikrein is represented by blue surface with the catalytic Ser-195 at the bottom of the reactive crevice indicated by red patch. Pro-MMP-9 is shown by orange and yellow ribbons corresponding to the pro and the catalytic domains, respectively. For simplicity, the fibronectin repeats are not shown. The first cleavage site of the pro-domain is marked by green arrow. The active site of MMP-9 contains the catalytic zinc (orange sphere) and the coordinated Cys-99, His-401, His-405, His-411 (sticks). Calcium ions are shown as green spheres.

\section{Time resolved XAS fitting results.}

Multiple Data-Set (MDS) Fits-The whole data set (17 spectra) was simultaneously fitted to a theoretical model calculated using FEFF $7^{5}$ using the MDS procedure ${ }^{1}$. This data analysis strategy, while employing several chemically and physically reasonable constraints between the fitting parameters, provides general trends in the changes in coordination number and metal-ligand bond distances. Multiple fitting of freezequenched samples was carried out by the following procedure: at each time point, the $\mathrm{k}^{2} \chi(\mathrm{k})$ data and theory were Fourier transformed into radial-space, where the non-linear least squares fits were performed concurrently, for 17 data sets ranging from 0 milliseconds (starting phase) to 1400 milliseconds, $2 \mathrm{~min}, 15 \mathrm{~min}$, and active MMP-9. The k-ranges and r-ranges in the fits were from 2 to $9-10 \AA^{-1}$ and from 1.1 to $2.1 \AA$, respectively. The same theoretical photoelectron scattering paths corresponding to the 
first shell Zn-N, Zn-O distances were fit to all 17 data sets simultaneously. To reduce the number of fit variables, we fixed the amplitude factor $\left(\mathrm{S}_{2}{ }_{2}\right)$ at 1 and the corrections to the photoelectron energy origin $\left(\Delta E_{0}\right)$ to be the same for all paths. The coordination number $(\mathrm{N}), \Delta \mathrm{R}$ and $\sigma 2$ of the $\mathrm{Zn}-\mathrm{O}$ and $\mathrm{Zn}-\mathrm{S}$ of all data sets were varied. This analysis resulted in the identification of three distinct spectral groups namely (I1-I3) that differed in coordination number and ligand identity. The full data set was than subjected to more refined analysis including PCA and RPA analyses ${ }^{1}$.

The following analysis included: 1 . Two dominating species were obtained using the PCA analysis. 2. The known species (pro-MMP-9 - the starting phase) was subtracted in $10 \%$ increments from the $\chi(k)$ data, such that for each time point, several residual spectra were generated. 3 . The residual spectra were automatically fed into FEFFIT ${ }^{6}$, using different combinations (number and species) of theoretical ligand contributions as calculated by FEFF ${ }^{5,7}$. 4. All results were sorted, and the best fits were manually refined. In our RPA procedure we treated the contribution from the three coordinating histidins with the same distances and Debye-Waller factors in order to obtain more degrees of freedom. Contributions from other ligands were varied in identity, number, and initial distance of the ligand from the zinc ion. The models are: a) $3 x Z n-N / O, 1 x Z n-$ N/O. b) 3xZn-N/O, 1xZn-S. c) 3xZn-N/O, 1xZn-S, 1xZn-N/O. d) 3xZn-N/O, 2xZn-N/O. e) $4 x Z n-N / O, 1 x Z n-S$. f) $3 x Z n-N / O, 1 x Z n-N / O, 2 x Z n-N / O$. Overall, each sample resulted in 60 RPA fits. Due to space limitations we present only fits with $\sigma^{2}<0.01 \AA^{2}$ and $\Delta \mathrm{R}<$ $10 \%$ of the ligand-metal distance. The fits are sorted by increasing order of $\chi^{2}$ values. The fits were than further tested for stability and refined using conventional EXAFS fitting procedures, and the best fit was chosen. The parameters of the best fit are presented, as well as its representation in $\mathrm{R}$ and back transformed $k$ space, where solid line represents the data and dotted line represents the fit.

In the RPA table, residuals refer to the percentage of the unknown species to be fitted. $\chi^{2}$ is the goodness of the fit. $\Delta \mathrm{E}_{0}$ is the correction to the energy origin. $\mathrm{R}$ is the distance from the zinc ion in $\AA$. $\sigma^{2}$ is the Debye-Waller factor. In cases that error values are absent it indicates that the distance values were fixed after they were guessed in a previous fit. Error values in the best-fit table are represented in parentheses and refer to the last digit mentioned. 


\section{$\underline{100 \mathrm{~ms}}$}

RPA:

\begin{tabular}{|c|c|c|c|c|c|c|c|c|c|c|}
\hline & & & \multicolumn{2}{|c|}{$3 \times \mathrm{Zn}-\mathrm{N} / O-\mathrm{I}$} & \multicolumn{2}{|c|}{$1 \times \mathrm{Zn}-\mathrm{S}$} & \multicolumn{2}{|c|}{$1 \times \mathrm{Zn}-\mathrm{N} / \mathrm{O}$ - II } & \multicolumn{2}{|c|}{ 1x Zn-N/O - III } \\
\hline Residual & $\chi^{2}$ & $\Delta \mathbf{E}_{0}$ & $\mathbf{R}[\AA]$ & $\sigma^{2}\left[\AA^{2}\right]$ & $\mathbf{R}[\AA ̊ \mathbf{A}]$ & $\sigma^{2}\left[\AA^{2}\right]$ & $\mathbf{R}[\AA]$ & $\sigma^{2}\left[\AA^{2}\right]$ & $\mathbf{R}[\AA]]$ & $\sigma^{2}\left[\AA^{2}\right]$ \\
\hline 70 & 104.57 & -3.98 & 2.00 & $-9.22 \mathrm{E}-06$ & & & 1.92 & 9E-07 & 1.92263 & 9E-07 \\
\hline 60 & 113.94 & -4.33 & 1.98 & $4.81 \mathrm{E}-06$ & & & 1.94 & $1.3 \mathrm{E}-06$ & 1.9424 & $1.3 \mathrm{E}-06$ \\
\hline 100 & 126.04 & 2.70 & 2.05 & 2.93E-03 & & & 1.99 & -1.76E-07 & & \\
\hline 70 & 130.37 & -0.10 & 2.00 & $-7.32 E-07$ & & & 1.98 & 3.88E-07 & & \\
\hline 100 & 139.46 & 6.34 & 2.05 & $1.99 \mathrm{E}-06$ & 2.46 & $1.76 \mathrm{E}-03$ & & & & \\
\hline 80 & 154.80 & -16.95 & 3.86 & $-5.08 E-07$ & 2.12 & 9.09E-05 & 1.88 & 1.04E-06 & & \\
\hline 80 & 164.50 & -0.35 & 2.03 & 1.02E-06 & 2.19 & 6.69E-03 & & & & \\
\hline 40 & 215.49 & -4.88 & 1.99 & 3.54E-07 & 2.17 & $-1.82 \mathrm{E}-05$ & & & & \\
\hline 30 & 218.01 & -1.20 & 2.04 & $-1.26 \mathrm{E}-05$ & 2.16 & $-1.86 \mathrm{E}-08$ & 1.97 & $-1.6 \mathrm{E}-06$ & 1.97099 & -1.6E-06 \\
\hline 30 & 229.34 & -0.11 & 2.03 & $3.48 \mathrm{E}-08$ & & & 1.98 & $-6.77 \mathrm{E}-07$ & 1.98056 & $-6.8 \mathrm{E}-07$ \\
\hline 30 & 240.47 & -1.44 & 2.04 & 9.95E-07 & 2.16 & 1.63E-07 & 1.98 & $-3 E-06$ & & \\
\hline 20 & 266.02 & -0.36 & 2.01 & 8.04E-07 & & & 1.98 & $2.04 \mathrm{E}-06$ & 1.97733 & $2 \mathrm{E}-06$ \\
\hline 20 & 285.41 & -3.80 & 1.98 & 5.77E-07 & 2.19 & -3.56E-06 & & & & \\
\hline
\end{tabular}

\section{Best fit:}

\begin{tabular}{|c|c|c|c|c|c|c|c|c|c|c|c|}
\hline & & & & $3 \times Z$ & N/O - I & & $\mathrm{Zn}-\mathrm{S}$ & $1 \times Z r$ & N/O - II & $1 \times \mathrm{Zn}-$ & /O- III \\
\hline Time (ms) & Residual & $\chi^{2}$ & $\Delta \mathbf{E}_{0}$ & $\mathbf{R}[\AA ̊]$ & $\sigma^{2}\left[\AA^{2}\right]$ & $\mathbf{R}[\AA]$ & $\sigma^{2}\left[\AA^{2}\right]$ & $\mathbf{R}[\AA]$ & $\sigma^{2}\left[\AA^{2}\right]$ & $\mathbf{R}[\AA]$ & $\sigma^{2}\left[\AA^{2}\right]$ \\
\hline 100 & 70 & 1.17 & -0.95 & 2.04(1) & $2.80 \mathrm{E}-03$ & $2.24(2)$ & 3.50E-04 & $2.04(1)$ & $2.80 \mathrm{E}-03$ & & \\
\hline
\end{tabular}

\section{$\underline{\text { Curve fitting analysis: }}$}

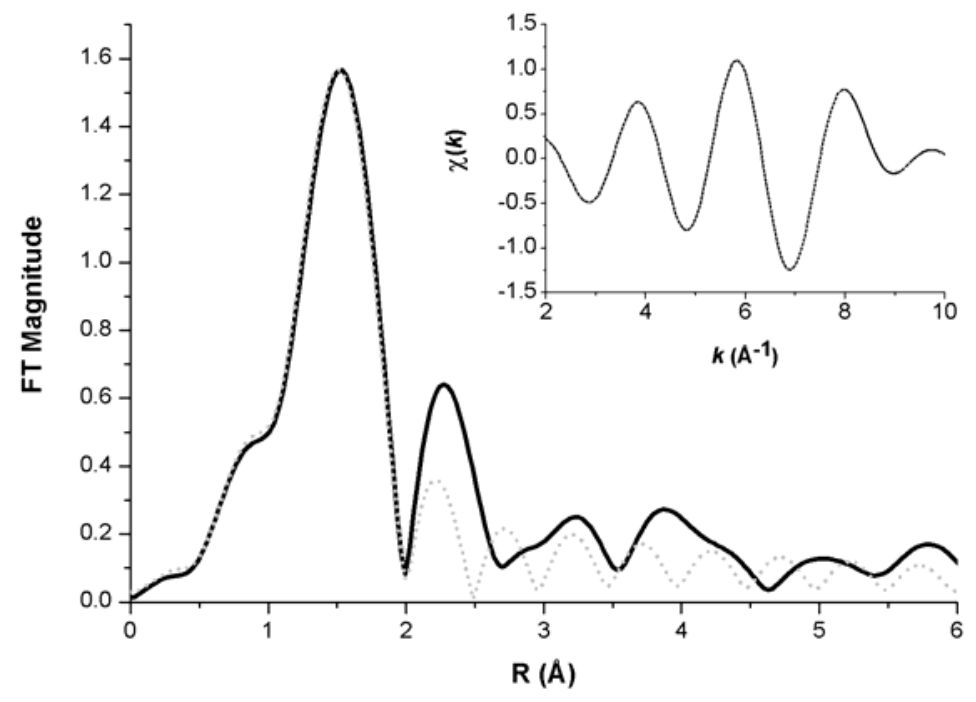


RPA:

$\underline{150 \mathrm{~ms}}$

\begin{tabular}{|c|c|c|c|c|c|c|c|c|c|c|}
\hline \multirow[b]{2}{*}{ Residual } & \multirow[b]{2}{*}{$\chi^{2}$} & \multirow[b]{2}{*}{$\Delta \mathbf{E}_{0}$} & \multicolumn{2}{|c|}{$3 \times \mathrm{Zn}-\mathrm{N} / \mathrm{O}$ - I } & \multicolumn{2}{|c|}{$1 \times \mathrm{Zn}-\mathrm{S}$} & \multicolumn{2}{|c|}{$1 \times \mathrm{Zn}-\mathrm{N} / \mathrm{O}$ - II } & \multicolumn{2}{|c|}{$1 \times \mathrm{Zn}-\mathrm{N} / \mathrm{O}-\mathrm{III}$} \\
\hline & & & $\mathbf{R}[\AA]$ & $\sigma^{2}\left[\AA^{2}\right]$ & $\mathbf{R}[\AA]$ & $\sigma^{2}\left[\AA^{2}\right]$ & $\mathbf{R}[\AA \mathbf{A}]$ & $\sigma^{2}\left[\AA^{2}\right]$ & $\mathbf{R}[\AA]$ & $\sigma^{2}\left[\AA^{2}\right]$ \\
\hline 90 & 16.39 & -13.57 & 1.93 & $1.52 \mathrm{E}-04$ & 2.26 & $-5.70 \mathrm{E}-04$ & 1.82 & $8.17 \mathrm{E}-06$ & & \\
\hline 100 & 16.82 & -12.92 & 1.93 & $4.83 \mathrm{E}-06$ & 2.27 & -1.09E-03 & 1.81 & $-4.00 \mathrm{E}-04$ & & \\
\hline 80 & 20.18 & -13.12 & 1.93 & $-2.20 \mathrm{E}-03$ & 2.27 & -1.61E-03 & 1.85 & $-7.70 \mathrm{E}-07$ & & \\
\hline 70 & 20.63 & -13.93 & 1.90 & 9.56E-04 & 2.26 & $-4.70 \mathrm{E}-07$ & 1.89 & \begin{tabular}{|l|}
$1.45 \mathrm{E}-04$ \\
\end{tabular} & & \\
\hline 100 & 42.40 & -12.29 & 1.91 & $-2.30 \mathrm{E}-07$ & 2.25 & 6.97E-04 & & & & \\
\hline 90 & 48.43 & -11.12 & 1.92 & $4.30 \mathrm{E}-08$ & 2.27 & $1.17 \mathrm{E}-03$ & & & & \\
\hline 80 & 64.78 & -10.65 & 1.92 & $1.87 \mathrm{E}-07$ & 2.27 & $2.71 \mathrm{E}-03$ & & & & \\
\hline 70 & 83.39 & -10.37 & 1.93 & $-6.10 \mathrm{E}-07$ & 2.28 & $3.49 \mathrm{E}-03$ & & & & \\
\hline 60 & 111.65 & -13.76 & 1.94 & $-6.19 \mathrm{E}-03$ & & & 1.89 & $1.82 \mathrm{E}-03$ & 1.89 & $1.82 \mathrm{E}-03$ \\
\hline 10 & 151.72 & -8.52 & 1.45 & $2.87 \mathrm{E}-07$ & & & 1.95 & $9.19 \mathrm{E}-07$ & 1.95 & $9.19 \mathrm{E}-07$ \\
\hline 70 & 160.44 & -9.78 & 1.92 & $2.49 \mathrm{E}-06$ & & & 2.01 & $5.26 \mathrm{E}-06$ & & \\
\hline 80 & 169.35 & -8.68 & 1.92 & $-2.20 \mathrm{E}-04$ & & & 2.02 & $-2.20 \mathrm{E}-06$ & & \\
\hline 10 & 171.86 & -4.86 & 1.46 & $-2.40 \mathrm{E}-07$ & & & 1.97 & \begin{tabular}{|l|}
$1.17 \mathrm{E}-07$ \\
\end{tabular} & & \\
\hline 90 & 177.86 & -7.48 & 1.93 & $-2.60 \mathrm{E}-04$ & & & 2.03 & -7.00E-07 & & \\
\hline 100 & 186.89 & -6.87 & 1.93 & 1.99E-04 & & & 2.04 & $-1.60 \mathrm{E}-06$ & & \\
\hline 80 & 200.36 & -7.55 & 1.95 & \begin{tabular}{|l|}
$1.44 \mathrm{E}-03$ \\
\end{tabular} & & & 2.13 & \begin{tabular}{|l|}
$4.63 \mathrm{E}-03$ \\
\end{tabular} & & \\
\hline
\end{tabular}

Best fit:

\begin{tabular}{|c|c|c|c|c|c|c|c|c|c|c|c|}
\hline & & & & $3 \times Z$ & N/O - I & & $\mathrm{Zn}-\mathrm{S}$ & $1 \times Z_{r}$ & N/O - II & $1 \times \mathrm{Zn}-$ & /O - III \\
\hline Time (ms) & Residual & $\chi^{2}$ & $\Delta \mathbf{E}_{0}$ & $\mathbf{R}[\AA]$ & $\sigma^{2}\left[\AA^{2}\right]$ & $\mathbf{R}[\AA]]$ & $\sigma^{2}\left[\AA^{2}\right]$ & $\mathbf{R}[\AA]$ & $\sigma^{2}\left[\AA^{2}\right]$ & $\mathbf{R}[\AA ̊]$ & $\sigma^{2}\left[\AA^{2}\right]$ \\
\hline 150 & 90 & 8.28 & 3.2 & $1.90(1)$ & $2.00 \mathrm{E}-03$ & $2.25(2)$ & $1.20 \mathrm{E}-03$ & $1.90(1)$ & $2.00 \mathrm{E}-03$ & & \\
\hline
\end{tabular}

\section{$\underline{\text { Curve fitting analysis: }}$}

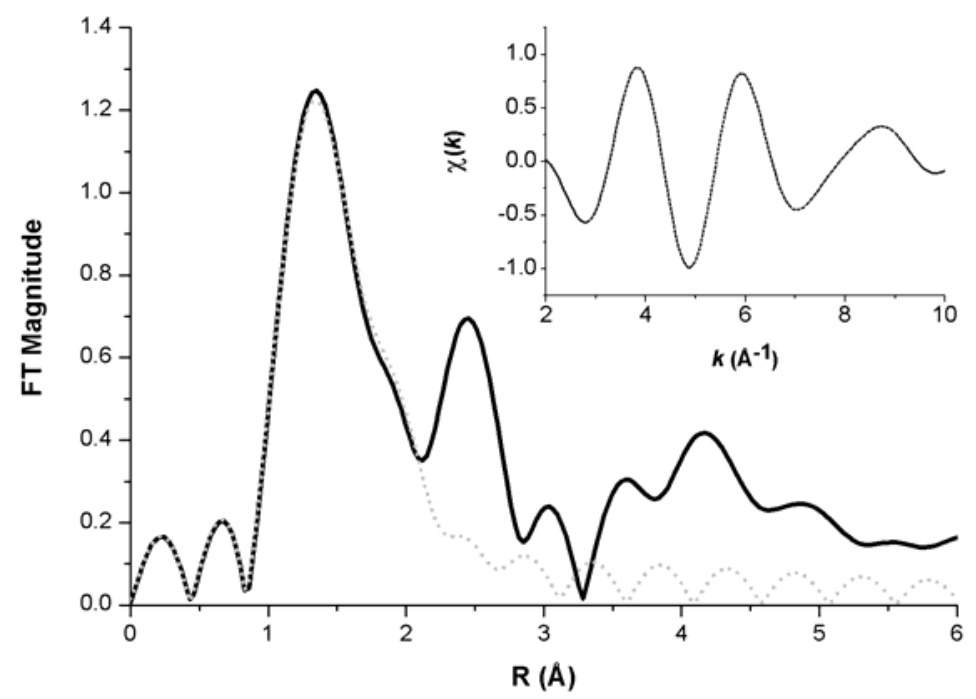




\section{$\underline{200 \mathrm{~ms}}$}

RPA:

\begin{tabular}{|c|c|c|c|c|c|c|c|c|c|c|}
\hline \multirow[b]{2}{*}{ Residual } & \multirow[b]{2}{*}{$\chi^{2}$} & \multirow[b]{2}{*}{$\Delta \mathbf{E}_{0}$} & \multicolumn{2}{|c|}{$3 \times \mathrm{Zn}-\mathrm{N} / \mathrm{O}$ - I } & \multicolumn{2}{|c|}{$1 \times \mathrm{Zn}-\mathrm{S}$} & \multicolumn{2}{|c|}{$1 \times \mathrm{Zn}-\mathrm{N} / \mathrm{O}$ - II } & \multicolumn{2}{|c|}{ 1x Zn-N/O - III } \\
\hline & & & $\mathbf{R}[\AA \mathbf{A}]$ & $\sigma^{2}\left[\AA^{2}\right]$ & $\mathbf{R}[\AA]$ & $\sigma^{2}\left[\AA^{2}\right]$ & $\mathbf{R}[\AA]$ & $\sigma^{2}\left[\AA^{2}\right]$ & $\mathbf{R}[\AA]$ & $\sigma^{2}\left[\AA^{2}\right]$ \\
\hline 100 & 60.40 & -3.80 & 1.99 & -1.16E-03 & 2.33 & $-2.24 \mathrm{E}-07$ & 1.91 & -0.00116 & & \\
\hline 90 & 60.91 & -7.62 & 1.96 & $2.74 \mathrm{E}-04$ & 2.30 & $-1.72 \mathrm{E}-06$ & 1.91 & 0.00027 & & \\
\hline 90 & 78.15 & -6.94 & 1.95 & $3.38 \mathrm{E}-07$ & 2.29 & $-1.99 \mathrm{E}-06$ & & & & \\
\hline 100 & 92.19 & -0.49 & 2.08 & 1.93E-06 & 2.38 & $2.61 \mathrm{E}-03$ & 1.91 & $1.93 \mathrm{E}-06$ & 1.91 & $1.93 \mathrm{E}-06$ \\
\hline 80 & 103.81 & -5.96 & 1.96 & $5.88 \mathrm{E}-08$ & 2.30 & $-4.30 \mathrm{E}-04$ & & & & \\
\hline 40 & 104.83 & 15.60 & 1.59 & $1.54 \mathrm{E}-06$ & & & 2.08 & $4.82 \mathrm{E}-06$ & & \\
\hline 70 & 110.01 & 14.48 & 1.59 & $3.09 \mathrm{E}-03$ & & & 2.04 & $1.01 \mathrm{E}-06$ & & \\
\hline 100 & 116.12 & -3.69 & 1.95 & $1.04 \mathrm{E}-06$ & & & 2.06 & $1.87 \mathrm{E}-06$ & & \\
\hline 100 & 121.39 & -2.79 & 1.97 & $-1.50 \mathrm{E}-04$ & & & 2.10 & $-7.1 \mathrm{E}-07$ & & \\
\hline 90 & 125.48 & -7.05 & 1.94 & $1.18 \mathrm{E}-07$ & & & 2.01 & $-1.79 \mathrm{E}-03$ & & \\
\hline 80 & 142.49 & 0.69 & 2.06 & $6.51 \mathrm{E}-07$ & 2.39 & $-9.59 \mathrm{E}-07$ & 1.91 & $6.5 \mathrm{E}-07$ & & \\
\hline 60 & 178.07 & -4.14 & 1.99 & $-9.26 \mathrm{E}-07$ & 2.32 & $3.06 \mathrm{E}-04$ & & & & \\
\hline 30 & 228.77 & -0.32 & 1.51 & $-2.02 \mathrm{E}-07$ & & & 1.99 & $5.8 \mathrm{E}-08$ & & \\
\hline
\end{tabular}

Best fit:

\begin{tabular}{|c|c|c|c|c|c|c|c|c|c|c|c|}
\hline \multirow[b]{2}{*}{ Time (ms) } & \multirow[b]{2}{*}{ Residual } & \multirow[b]{2}{*}{$\chi^{2}$} & \multirow[b]{2}{*}{$\Delta \mathbf{E}_{0}$} & \multicolumn{2}{|c|}{$3 \times \mathrm{Zn}-\mathrm{N} / \mathrm{O}$ - I } & \multicolumn{2}{|c|}{$1 \times \mathrm{Zn}-\mathrm{S}$} & \multicolumn{2}{|c|}{$1 \times \mathrm{Zn}-\mathrm{N} / \mathrm{O}-\mathrm{II}$} & \multicolumn{2}{|c|}{ 1x Zn-N/O - III } \\
\hline & & & & $\mathbf{R}[\AA]$ & $\sigma^{2}\left[\AA^{2}\right]$ & $\mathbf{R}[\AA]$ & $\sigma^{2}\left[\AA^{2}\right]$ & $\mathbf{R}[\AA ̊]$ & $\sigma^{2}\left[\AA^{2}\right]$ & $\mathbf{R}[\AA]$ & $\sigma^{2}\left[\AA^{2}\right]$ \\
\hline 200 & 80 & 3.48 & 0.86 & $2.03(3)$ & $5.20 \mathrm{E}-03$ & $2.30(4)$ & $3.70 \mathrm{E}-03$ & $2.03(3)$ & $5.20 \mathrm{E}-03$ & & \\
\hline
\end{tabular}

\section{Curve fitting analysis:}

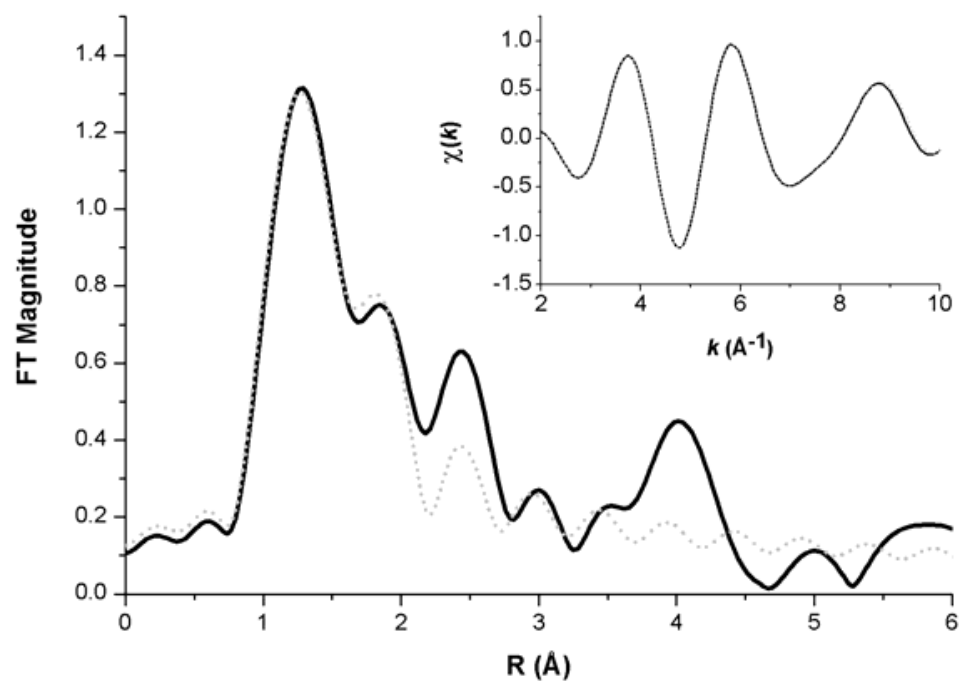




\section{0ms}

RPA:

\begin{tabular}{|c|c|c|c|c|c|c|c|c|c|c|}
\hline \multirow[b]{2}{*}{ Residual } & \multirow[b]{2}{*}{$\chi^{2}$} & \multirow[b]{2}{*}{$\Delta \mathbf{E}_{0}$} & \multicolumn{2}{|c|}{$3 \times \mathrm{Zn}-\mathrm{N} / \mathrm{O}$ - I } & \multicolumn{2}{|c|}{$1 \times \mathrm{Zn}-\mathrm{S}$} & \multicolumn{2}{|c|}{$1 \times \mathrm{Zn}-\mathrm{N} / \mathrm{O}$ - II } & \multicolumn{2}{|c|}{$1 \times \mathrm{Zn}-\mathrm{N} / \mathrm{O}-\mathrm{III}$} \\
\hline & & & $\mathbf{R}[\AA]$ & $\sigma^{2}\left[\AA^{2}\right]$ & $\mathbf{R}[\mathbf{A}]$ & $\sigma^{2}\left[\AA^{2}\right]$ & $\mathbf{R}[\AA]$ & $\sigma^{2}\left[\AA^{2}\right]$ & $\mathbf{R}[\AA]$ & $\sigma^{2}\left[\AA^{2}\right]$ \\
\hline 40 & 21.33 & -4.43 & 2.01 & $6.05 \mathrm{E}-05$ & 2.32 & $-2.60 \mathrm{E}-07$ & 1.97 & $-1.00 \mathrm{E}-04$ & 1.97 & $-1.00 \mathrm{E}-04$ \\
\hline 50 & 23.43 & -4.65 & 1.99 & \begin{tabular}{|l|}
$1.69 \mathrm{E}-06$ \\
\end{tabular} & 2.30 & $-6.90 \mathrm{E}-07$ & 1.99 & $1.78 \mathrm{E}-06$ & & \\
\hline 100 & 31.96 & -3.58 & 2.00 & $6.00 \mathrm{E}-03$ & 2.27 & $-3.70 \mathrm{E}-03$ & 1.97 & $2.49 \mathrm{E}-03$ & & \\
\hline 90 & 32.58 & -5.02 & 1.98 & $1.28 \mathrm{E}-03$ & 2.26 & $-1.80 \mathrm{E}-07$ & & & & \\
\hline 80 & 33.53 & -4.69 & 1.99 & $5.28 \mathrm{E}-04$ & 2.27 & 1.17E-07 & & & & \\
\hline 100 & 33.59 & -4.95 & 1.98 & \begin{tabular}{|l|}
$1.85 \mathrm{E}-03$ \\
\end{tabular} & 2.26 & $3.32 \mathrm{E}-07$ & & & & \\
\hline 70 & 37.13 & -4.95 & 1.99 & $1.14 \mathrm{E}-07$ & 2.27 & $8.55 \mathrm{E}-07$ & & & & \\
\hline 40 & 50.53 & -4.76 & 2.00 & $-1.20 \mathrm{E}-07$ & 2.30 & $-1.80 \mathrm{E}-06$ & 1.98 & 4.18E-07 & & \\
\hline 60 & 53.95 & -5.22 & 1.99 & $-3.60 \mathrm{E}-07$ & 2.27 & $-1.40 \mathrm{E}-06$ & & & & \\
\hline 50 & 88.95 & -5.36 & 2.00 & $1.57 \mathrm{E}-06$ & 2.27 & $2.49 \mathrm{E}-06$ & & & & \\
\hline 60 & 113.10 & -2.23 & 2.04 & $9.98 \mathrm{E}-05$ & & & 1.97 & 2.81E-06 & & \\
\hline 70 & 121.60 & -1.78 & 2.02 & $1.33 \mathrm{E}-03$ & & & 2.01 & 4.32E-04 & & \\
\hline 80 & 122.41 & -1.35 & 2.05 & $1.02 \mathrm{E}-03$ & & & 1.95 & $-2.60 \mathrm{E}-05$ & & \\
\hline 40 & 131.05 & -6.27 & 2.00 & 2.35E-07 & 2.26 & $-5.60 \mathrm{E}-06$ & & & & \\
\hline 90 & 137.36 & -0.92 & 2.05 & $1.53 \mathrm{E}-03$ & & & 1.95 & $-1.30 \mathrm{E}-06$ & & \\
\hline 100 & 158.49 & -0.70 & 2.05 & $2.22 \mathrm{E}-03$ & & & 1.96 & $7.48 \mathrm{E}-04$ & & \\
\hline 20 & 186.36 & -5.64 & 2.01 & \begin{tabular}{|l}
$-1.60 \mathrm{E}-07$ \\
\end{tabular} & & & 2.00 & $-1.50 \mathrm{E}-08$ & 2.00 & $-1.50 \mathrm{E}-08$ \\
\hline 30 & 211.43 & -3.55 & 2.03 & $-4.40 \mathrm{E}-08$ & 2.32 & $2.24 \mathrm{E}-03$ & & & & \\
\hline 20 & 267.21 & -3.26 & 2.02 & 5.33E-07 & 2.35 & 3.67E-04 & & & & \\
\hline
\end{tabular}

$\underline{B e s t}$ fit:

\begin{tabular}{|c|c|c|c|c|c|c|c|c|c|c|c|}
\hline \multirow[b]{2}{*}{ Time (ms) } & \multirow[b]{2}{*}{ Residual } & \multirow[b]{2}{*}{$\chi^{2}$} & \multirow[b]{2}{*}{$\Delta \mathbf{E}_{0}$} & \multicolumn{2}{|c|}{$3 \times \mathrm{Zn}-\mathrm{N} / \mathrm{O}-\mathrm{I}$} & \multicolumn{2}{|c|}{$1 \times \mathrm{Zn}-\mathrm{S}$} & \multicolumn{2}{|c|}{$1 \times \mathrm{Zn}-\mathrm{N} / \mathrm{O}$ - II } & \multicolumn{2}{|c|}{ 1x Zn-N/O - III } \\
\hline & & & & $\mathbf{R}[\AA]$ & $\sigma^{2}\left[\AA^{2}\right]$ & $\mathbf{R}[\AA]$ & $\sigma^{2}\left[\AA^{2}\right]$ & $\mathbf{R}[\AA]$ & $\sigma^{2}\left[\AA^{2}\right]$ & $\mathbf{R}[\AA]$ & $\sigma^{2}\left[\AA^{2}\right]$ \\
\hline 250 & 60 & 24.40 & -3.52 & $2.01(1)$ & $3.50 \mathrm{E}-03$ & $2.31(2)$ & 4.70E-03 & $1.99(1)$ & $3.50 \mathrm{E}-03$ & & \\
\hline
\end{tabular}

\section{Curve fitting analysis:}

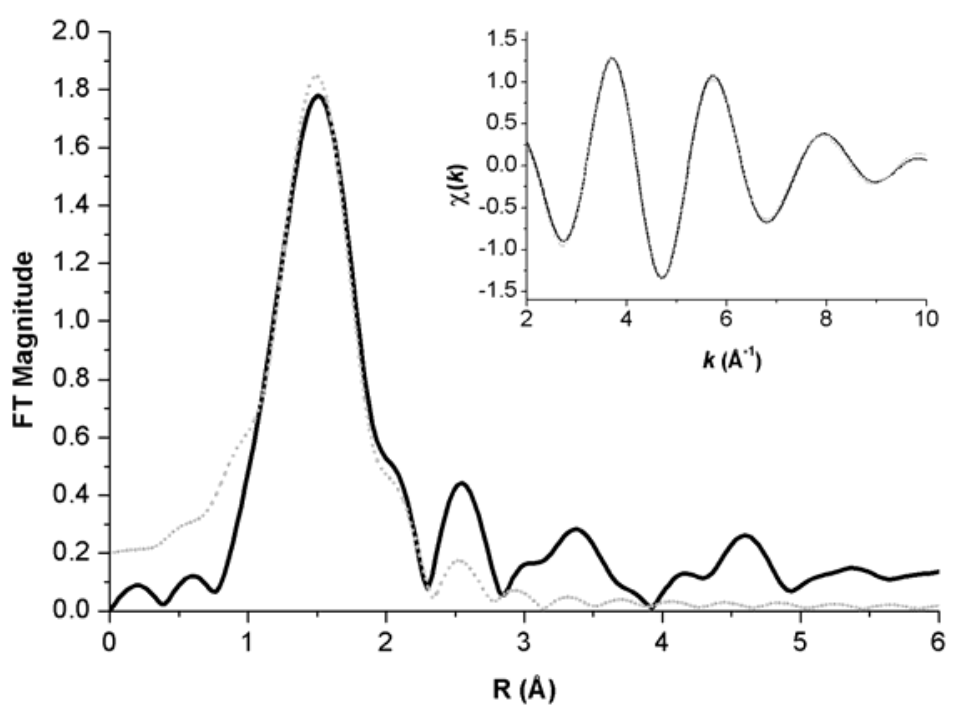


RPA:

$\underline{300 \mathrm{~ms}}$

\begin{tabular}{|c|c|c|c|c|c|c|c|c|c|c|}
\hline & & & \multicolumn{2}{|c|}{$3 \times \mathrm{Zn}-\mathrm{N} / \mathrm{O}-\mathrm{I}$} & \multicolumn{2}{|c|}{$1 \times \mathrm{Zn}-\mathrm{S}$} & \multicolumn{2}{|c|}{$1 \times \mathrm{Zn}-\mathrm{N} / O-\mathrm{II}$} & \multicolumn{2}{|c|}{$1 \times \mathrm{Zn}-\mathrm{N} / \mathrm{O}-\mathrm{III}$} \\
\hline Residual & $\chi^{2}$ & $\Delta \mathbf{E}_{0}$ & $\mathbf{R}[\AA]$ & $\sigma^{2}\left[\AA^{2}\right]$ & $\mathbf{R}[\AA]$ & $\sigma^{2}\left[\AA^{2}\right]$ & $\mathbf{R}[\AA]$ & $\sigma^{2}\left[\AA^{2}\right]$ & $\mathbf{R}[\AA]$ & $\sigma^{2}\left[\AA^{2}\right]$ \\
\hline 100 & 13.37 & 3.46 & 2.02 & $6.22 \mathrm{E}-03$ & & & 2.05 & $6.22 \mathrm{E}-03$ & & \\
\hline 90 & 15.39 & 2.66 & 2.01 & \begin{tabular}{|l|}
$4.35 \mathrm{E}-03$ \\
\end{tabular} & & & 2.07 & $4.35 \mathrm{E}-03$ & & \\
\hline 100 & 15.89 & 3.39 & 2.04 & $6.29 \mathrm{E}-03$ & & & 2.04 & $6.28 \mathrm{E}-03$ & & \\
\hline 80 & 19.70 & 1.94 & 2.00 & $1.77 \mathrm{E}-03$ & & & 2.09 & 0.00177 & & \\
\hline 70 & 24.84 & 0.78 & 1.99 & $3.13 \mathrm{E}-07$ & & & 2.09 & $3.13 \mathrm{E}-07$ & & \\
\hline 60 & 26.31 & -4.40 & 1.97 & $-4.06 \mathrm{E}-06$ & 2.17 & 9.75E-03 & 2.07 & $-4.1 \mathrm{E}-06$ & & \\
\hline 60 & 29.97 & -1.50 & 1.98 & \begin{tabular}{|l|}
$9.96 \mathrm{E}-07$ \\
\end{tabular} & & & 2.05 & $9.96 \mathrm{E}-07$ & & \\
\hline 50 & 32.01 & -4.93 & 1.98 & $-6.18 \mathrm{E}-08$ & & & 1.96 & $-6.18 \mathrm{E}-08$ & & \\
\hline 60 & 32.35 & -1.78 & 2.01 & \begin{tabular}{|c|}
$-1.25 \mathrm{E}-03$ \\
\end{tabular} & & & 1.98 & $-2.53 \mathrm{E}-06$ & & \\
\hline 40 & 61.10 & -5.50 & 2.05 & $-1.86 \mathrm{E}-07$ & 2.14 & $-4.53 \mathrm{E}-07$ & 1.94 & $-1.9 \mathrm{E}-07$ & & \\
\hline 30 & 83.23 & -5.37 & 2.02 & $-3.24 \mathrm{E}-07$ & 2.15 & $-4.10 \mathrm{E}-07$ & 1.97 & $-3.24 \mathrm{E}-07$ & & \\
\hline 20 & 172.52 & -2.03 & 2.05 & $2.56 \mathrm{E}-07$ & 2.17 & $-5.83 \mathrm{E}-09$ & 1.98 & $2.56 \mathrm{E}-07$ & & \\
\hline
\end{tabular}

$\underline{\text { Best Fit }}$

\begin{tabular}{|c|c|c|c|c|c|c|c|c|c|c|c|}
\hline & & & & \multicolumn{2}{|c|}{$3 \times \mathrm{Zn}-\mathrm{N} / \mathrm{O}-\mathrm{I}$} & \multicolumn{2}{|c|}{$1 \times \mathrm{Zn}-\mathrm{S}$} & \multicolumn{2}{|c|}{$1 \times \mathrm{Zn}-\mathrm{N} / \mathrm{O}$ - II } & \multicolumn{2}{|c|}{ 1x Zn-N/O - III } \\
\hline Time (ms) & Residual & $\chi^{2}$ & $\Delta \mathbf{E}_{0}$ & $\mathbf{R}[\mathbf{A}]$ & $\sigma^{2}\left[\AA^{2}\right]$ & $\mathbf{R}[\mathbf{A}]$ & $\sigma^{2}\left[\AA^{2}\right]$ & $\mathbf{R}[\mathbf{A}]$ & $\sigma^{2}\left[\AA^{2}\right]$ & $\mathbf{R}[\AA]$ & $\sigma^{2}\left[\AA^{2}\right]$ \\
\hline 300 & 70 & 10.80 & 1.37 & $2.00(1)$ & 3.00E-03 & & & $1.99(1)$ & 3.00E-03 & & \\
\hline
\end{tabular}

\section{$\underline{\text { Curve fitting analysis }}$}

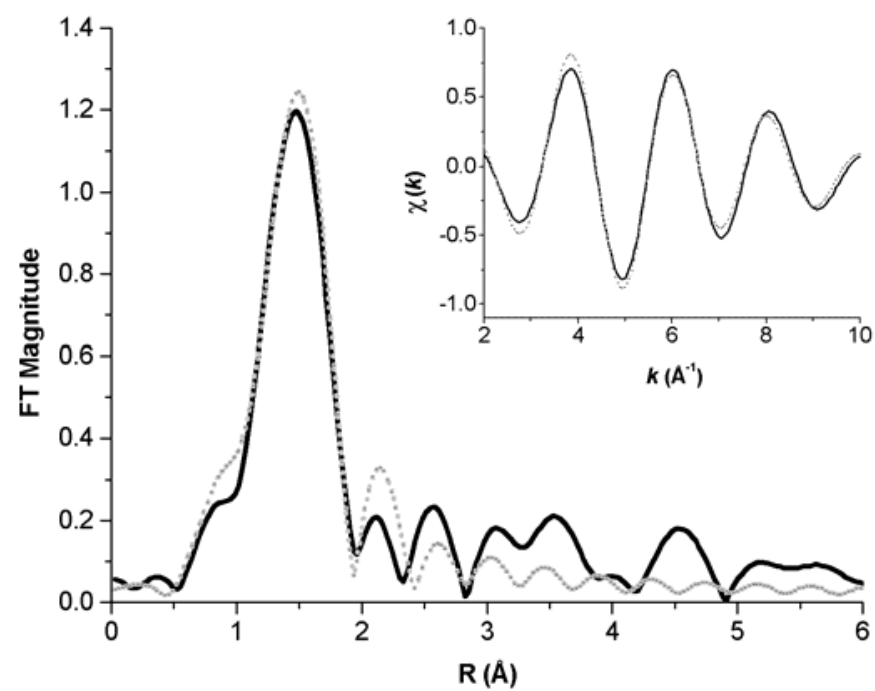




\section{$\underline{400 \mathrm{~ms}}$}

RPA:

\begin{tabular}{|c|c|c|c|c|c|c|c|c|c|c|}
\hline & & & \multicolumn{2}{|c|}{$3 \times \mathrm{Zn}-\mathrm{N} / \mathrm{O}-\mathrm{I}$} & \multicolumn{2}{|c|}{$1 \times \mathrm{Zn}-\mathrm{S}$} & \multicolumn{2}{|c|}{$1 \times \mathrm{Zn}-\mathrm{N} / \mathrm{O}-\mathrm{II}$} & \multicolumn{2}{|c|}{ 1x Zn-N/O - III } \\
\hline Residual & $\chi^{2}$ & $\Delta \mathbf{E}_{0}$ & $\mathbf{R}[\AA]$ & $\sigma^{2}\left[\AA^{2}\right]$ & $\mathbf{R}[\mathbf{A}]$ & $\sigma^{2}\left[\AA^{2}\right]$ & $\mathbf{R}[\AA]$ & $\sigma^{2}\left[\AA^{2}\right]$ & $\mathbf{R}[\AA]$ & $\sigma^{2}\left[\AA^{2}\right]$ \\
\hline 100 & 34.75 & 0.65 & 1.97 & $1.91 \mathrm{E}-03$ & & & 2.13 & $2.40 \mathrm{E}-03$ & 2.13 & $2.40 \mathrm{E}-03$ \\
\hline 50 & 51.62 & 3.65 & 2.13 & 4.32E-03 & & & 2.07 & $-9.20 \mathrm{E}-07$ & & \\
\hline 60 & 65.26 & 3.17 & 2.11 & $5.24 \mathrm{E}-03$ & & & 2.07 & 6.66E-07 & & \\
\hline 80 & 71.50 & 0.78 & 1.99 & $3.70 \mathrm{E}-03$ & & & 2.14 & $1.51 \mathrm{E}-06$ & & \\
\hline 100 & 79.49 & 0.03 & 1.98 & $3.38 \mathrm{E}-03$ & & & 2.12 & $6.38 \mathrm{E}-07$ & & \\
\hline 70 & 80.23 & -1.85 & 1.97 & 4.19E-03 & & & 2.10 & $2.50 \mathrm{E}-06$ & & \\
\hline 90 & 81.26 & -1.07 & 1.97 & $2.44 \mathrm{E}-03$ & & & 2.13 & $1.65 \mathrm{E}-06$ & & \\
\hline 80 & 90.33 & 2.19 & 2.09 & $5.33 \mathrm{E}-03$ & & & 2.05 & $1.27 \mathrm{E}-06$ & & \\
\hline 70 & 93.55 & -2.66 & 2.05 & $3.39 \mathrm{E}-03$ & & & 1.99 & $-9.50 \mathrm{E}-07$ & & \\
\hline 90 & 98.49 & 1.73 & 2.09 & $4.83 \mathrm{E}-03$ & & & 2.04 & $-1.40 \mathrm{E}-06$ & & \\
\hline 100 & 165.35 & -4.40 & 2.02 & $2.77 \mathrm{E}-03$ & & & 1.97 & $-1.60 \mathrm{E}-07$ & & \\
\hline
\end{tabular}

Best fit:

\begin{tabular}{|c|c|c|c|c|c|c|c|c|c|c|c|}
\hline \multirow[b]{2}{*}{ Time (ms) } & \multirow[b]{2}{*}{ Residual } & \multirow[b]{2}{*}{$\chi^{2}$} & \multirow[b]{2}{*}{$\Delta \mathbf{E}_{0}$} & \multicolumn{2}{|c|}{$3 \times \mathrm{Zn}-\mathrm{N} / \mathrm{O}-\mathrm{I}$} & \multicolumn{2}{|c|}{$1 \times \mathrm{Zn}-\mathrm{S}$} & \multicolumn{2}{|c|}{$1 \times \mathrm{Zn}-\mathrm{N} / \mathrm{O}$ - II } & \multicolumn{2}{|c|}{ 1x Zn-N/O - III } \\
\hline & & & & $\mathbf{R}[\AA ̊ \AA]$ & $\sigma^{2}\left[\AA^{2}\right]$ & $\mathbf{R}[\AA \mathbf{A}]$ & $\sigma^{2}\left[\AA^{2}\right]$ & $\mathbf{R}[\AA]$ & $\sigma^{2}\left[\AA^{2}\right]$ & $\mathbf{R}[\AA]$ & $\sigma^{2}\left[\AA^{2}\right]$ \\
\hline 400 & 60 & 0.12 & 2.12 & $2.20(1)$ & 4.20E-03 & & & $1.96(1)$ & $9.00 \mathrm{E}-04$ & & \\
\hline
\end{tabular}

\section{Curve Fitting analysis:}

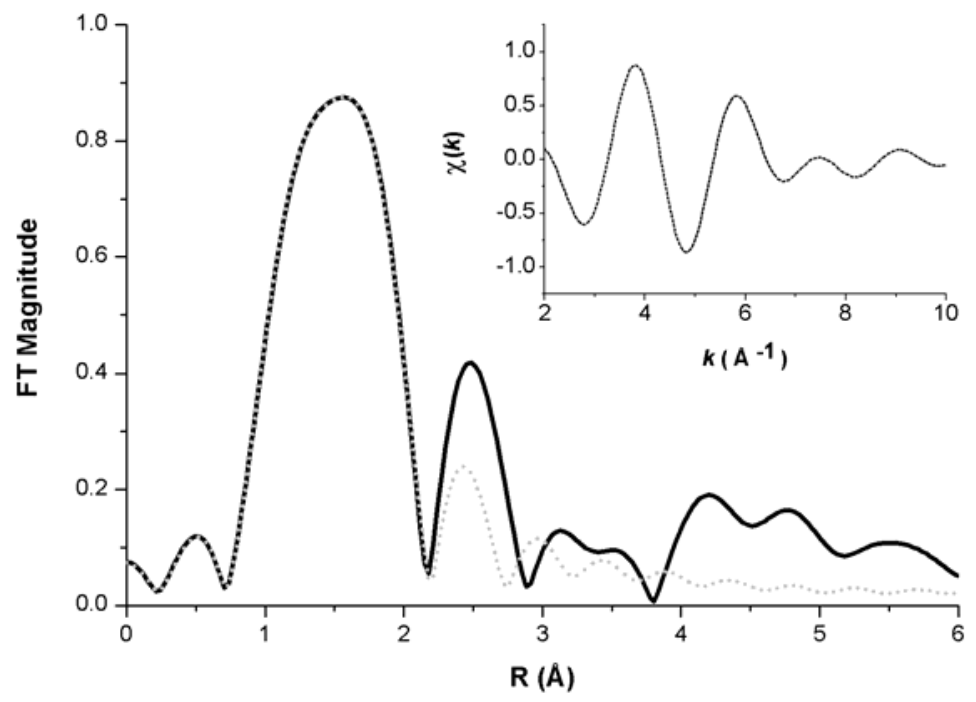


RPA:

$\underline{500 \mathrm{~ms}}$

\begin{tabular}{|c|c|c|c|c|c|c|c|c|c|c|}
\hline \multirow[b]{2}{*}{ Residual } & \multirow[b]{2}{*}{$\chi^{2}$} & \multirow[b]{2}{*}{$\Delta \mathbf{E}_{0}$} & \multicolumn{2}{|c|}{$3 \times \mathrm{Zn}-\mathrm{N} / \mathrm{O}-\mathrm{I}$} & \multicolumn{2}{|c|}{$1 \times \mathrm{Zn}-\mathrm{S}$} & \multicolumn{2}{|c|}{$1 \times \mathrm{Zn}-\mathrm{N} / \mathrm{O}$ - II } & \multicolumn{2}{|c|}{ 1x Zn-N/O - III } \\
\hline & & & $\mathbf{R}[\AA]$ & $\sigma^{2}\left[\AA^{2}\right]$ & $\mathbf{R}[\mathbf{A}]$ & $\sigma^{2}\left[\AA^{2}\right]$ & $\mathbf{R}[\AA]$ & $\sigma^{2}\left[\AA^{2}\right]$ & $\mathbf{R}[\AA]$ & $\sigma^{2}\left[\AA^{2}\right]$ \\
\hline 100 & 2.45 & 2.74 & 2.00 & $1.89 \mathrm{E}-03$ & & & 2.11 & $2.70 \mathrm{E}-03$ & & \\
\hline 90 & 3.05 & 2.37 & 2.00 & \begin{tabular}{|l|}
$1.60 \mathrm{E}-06$ \\
\end{tabular} & & & 2.12 & $1.36 \mathrm{E}-03$ & & \\
\hline 80 & 3.81 & 0.78 & 1.99 & $1.32 \mathrm{E}-06$ & & & 2.09 & $5.49 \mathrm{E}-03$ & & \\
\hline 70 & 4.85 & -1.60 & 1.98 & $-1.42 E-06$ & & & 2.02 & $4.31 \mathrm{E}-03$ & & \\
\hline 70 & 5.01 & -1.81 & 1.99 & $-5.82 \mathrm{E}-06$ & & & 2.02 & 4.45E-03 & & \\
\hline 60 & 5.56 & -6.17 & 2.01 & 4.02E-03 & 2.14 & $6.18 \mathrm{E}-03$ & 1.95 & 1.61E-07 & & \\
\hline 60 & 6.01 & -2.79 & 2.00 & $6.22 \mathrm{E}-06$ & & & 1.95 & $-3.3 \mathrm{E}-06$ & & \\
\hline 60 & 8.36 & -7.66 & 1.98 & -2.92E-07 & 2.16 & $2.29 \mathrm{E}-03$ & & & & \\
\hline 50 & 8.42 & -7.26 & 1.97 & $-4.01 \mathrm{E}-09$ & & & 1.94 & 0.00132 & 1.94091 & 0.00132 \\
\hline 50 & 8.94 & -2.36 & 2.01 & -3.26E-06 & 2.09 & -3.39E-07 & 2.03 & $1.94 \mathrm{E}-03$ & 2.02701 & 0.00194 \\
\hline 60 & 9.19 & -4.43 & 1.99 & -1.01E-07 & & & 1.95 & 0.00792 & 1.94705 & 0.00792 \\
\hline 70 & 9.73 & -2.60 & 2.01 & $1.30 \mathrm{E}-06$ & 2.18 & $7.82 \mathrm{E}-03$ & & & & \\
\hline 50 & 12.57 & -4.51 & 2.05 & $3.13 \mathrm{E}-03$ & 2.14 & $-4.18 \mathrm{E}-07$ & 1.95 & $9.38 \mathrm{E}-08$ & & \\
\hline 60 & 13.67 & -0.11 & 2.03 & 6.81E-07 & & & 1.97 & $-2.67 \mathrm{E}-07$ & & \\
\hline 40 & 14.76 & -5.17 & 1.98 & $2.12 \mathrm{E}-09$ & & & 1.96 & $4.51 \mathrm{E}-07$ & 1.95972 & $4.5 \mathrm{E}-07$ \\
\hline 50 & 15.28 & -2.66 & 1.98 & \begin{tabular}{|l|}
$8.60 \mathrm{E}-08$ \\
\end{tabular} & & & 1.98 & $-4.9 \mathrm{E}-09$ & & \\
\hline 50 & 18.72 & -2.88 & 2.00 & $-4.79 \mathrm{E}-08$ & & & 1.98 & $-5.25 \mathrm{E}-07$ & & \\
\hline 40 & 34.27 & -7.35 & 1.98 & $3.03 \mathrm{E}-07$ & 2.17 & -4.06E-08 & & & & \\
\hline 40 & 38.39 & -1.72 & 2.01 & \begin{tabular}{|l|}
$7.02 \mathrm{E}-08$ \\
\end{tabular} & & & 1.97 & 9.23E-08 & & \\
\hline 30 & 42.95 & -3.68 & 1.99 & \begin{tabular}{|l|}
$-9.45 E-08$ \\
\end{tabular} & & & 1.97 & $-1.13 \mathrm{E}-07$ & 1.97 & $-1.13 \mathrm{E}-0$ \\
\hline 30 & 67.83 & -6.99 & 1.99 & \begin{tabular}{|l|}
$-6.6 \mathrm{E}-08$ \\
\end{tabular} & 2.17 & $-4 \mathrm{E}-07$ & & & & \\
\hline 20.00 & 88 & -3.07 & 1.99 & 9.3E-09 & & & 1.97 & $-2.3 \mathrm{E}-08$ & 1.97253 & $-2.3 \mathrm{E}-08$ \\
\hline
\end{tabular}

Best fit:

\begin{tabular}{|c|c|c|c|c|c|c|c|c|c|c|c|}
\hline \multirow[b]{2}{*}{ Time (ms) } & \multirow[b]{2}{*}{ Residual } & \multirow[b]{2}{*}{$\chi^{2}$} & \multirow[b]{2}{*}{$\Delta \mathbf{E}_{0}$} & \multicolumn{2}{|c|}{$3 \times \mathrm{Zn}-\mathrm{N} / \mathrm{O}-\mathrm{I}$} & \multicolumn{2}{|c|}{$1 \times \mathrm{Zn}-\mathrm{S}$} & \multicolumn{2}{|c|}{1 x Zn-N/O - II } & \multicolumn{2}{|c|}{ 1x Zn-N/O - III } \\
\hline & & & & $\mathbf{R}[\AA]$ & $\sigma^{2}\left[\AA^{2}\right]$ & $\mathbf{R}[\AA]$ & $\sigma^{2}\left[\AA^{2}\right]$ & $\mathbf{R}[\AA ̊ \AA]$ & $\sigma^{2}\left[\AA^{2}\right]$ & $\mathbf{R}[\AA ̊]$ & $\sigma^{2}\left[\AA^{2}\right]$ \\
\hline 500 & 90 & 0.13 & 0.33 & $2.05(1)$ & 1.30E-04 & & & $1.90(3)$ & 1.30E-04 & & \\
\hline
\end{tabular}

\section{$\underline{\text { Curve fitting analysis }}$}

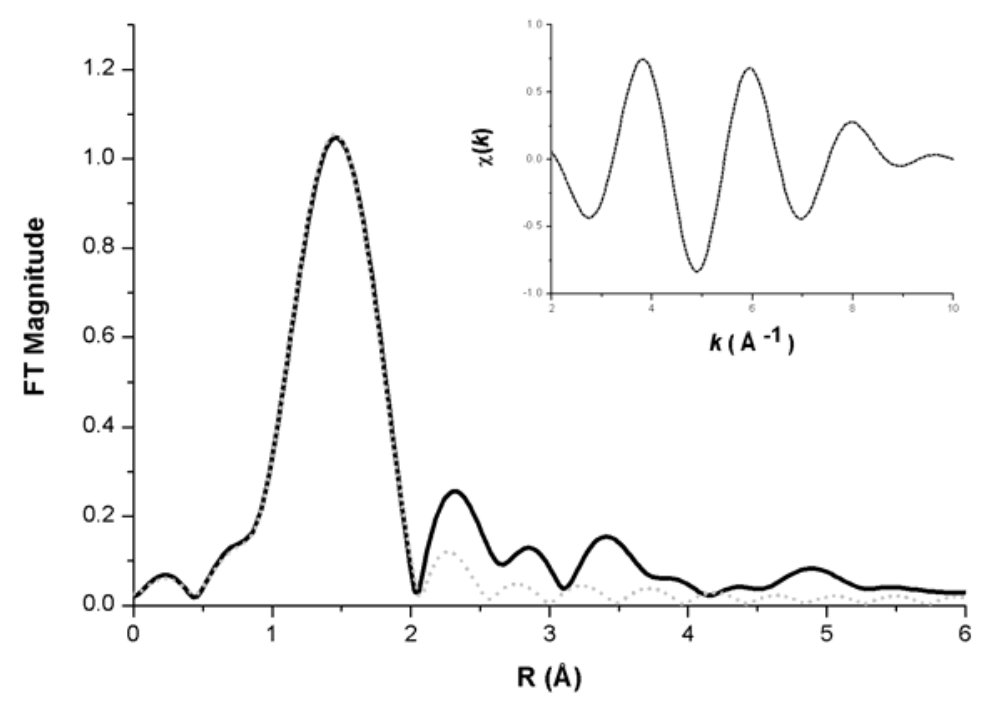


RPA:

$\underline{600 \mathrm{~ms}}$

\begin{tabular}{|c|c|c|c|c|c|c|c|c|c|c|}
\hline & & & \multicolumn{2}{|c|}{$3 \times \mathrm{Zn}-\mathrm{N} / \mathrm{O}-\mathrm{I}$} & \multicolumn{2}{|c|}{$1 \times \mathrm{Zn}-\mathrm{S}$} & \multicolumn{2}{|c|}{$1 \times \mathrm{Zn}-\mathrm{N} / \mathrm{O}$ - II } & \multicolumn{2}{|c|}{ 1x Zn-N/O - III } \\
\hline Residual & $\chi^{2}$ & $\Delta \mathrm{E}_{0}$ & $\mathbf{R}[\AA]$ & $\sigma^{2}\left[\AA^{2}\right]$ & $\mathbf{R}[\mathbf{A}]$ & $\sigma^{2}\left[\AA^{2}\right]$ & $\mathbf{R}[\AA]$ & $\sigma^{2}\left[\AA^{2}\right]$ & $\mathbf{R}[\AA]$ & $\sigma^{2}\left[\AA^{2}\right]$ \\
\hline 90 & 51.66 & 4.08 & 2.09 & $-2.48 \mathrm{E}-03$ & & & 1.93 & $1.58 \mathrm{E}-06$ & & \\
\hline 80 & 53.98 & 2.76 & 2.07 & \begin{tabular}{|l|}
$-1.63 \mathrm{E}-03$ \\
\end{tabular} & & & 1.93 & $7.50 \mathrm{E}-08$ & & \\
\hline 40 & 57.20 & -4.78 & 1.99 & $1.86 \mathrm{E}-05$ & & & 1.97 & $-3.41 E-06$ & 1.97 & $-3.41 \mathrm{E}-06$ \\
\hline 60 & 62.97 & 0.19 & 2.01 & $4.96 \mathrm{E}-08$ & 2.34 & $4.15 \mathrm{E}-03$ & & & & \\
\hline 60 & 63.29 & -0.69 & 2.02 & $1.71 \mathrm{E}-03$ & & & 1.97 & $7.03 \mathrm{E}-04$ & & \\
\hline 80 & 64.74 & 2.18 & 2.06 & $2.20 \mathrm{E}-03$ & & & 1.96 & $-2.1 \mathrm{E}-06$ & 1.9572 & $-2.1 \mathrm{E}-06$ \\
\hline 50 & 66.98 & -2.26 & 2.00 & $4.25 \mathrm{E}-06$ & & & 1.98 & $-1.09 \mathrm{E}-06$ & & \\
\hline 50 & 74.24 & -1.90 & 2.01 & -2.13E-06 & & & 2.00 & \begin{tabular}{|l|}
$-3 E-07$ \\
\end{tabular} & & \\
\hline 100 & 76.01 & 4.12 & 2.03 & 6.09E-03 & & & 2.06 & 4.47E-03 & & \\
\hline 100 & 84.40 & 0.98 & 2.05 & 6.86E-04 & & & 1.90 & $-4.55 \mathrm{E}-08$ & & \\
\hline 50 & 89.41 & -2.31 & 2.01 & -7.86E-07 & 2.26 & 4.81E-03 & & & & \\
\hline 40 & 92.45 & -2.08 & 2.00 & 4.74E-07 & & & 1.99 & $-1.70 \mathrm{E}-07$ & & \\
\hline 40 & 118.27 & -0.17 & 2.04 & \begin{tabular}{|l|}
$-4.30 \mathrm{E}-07$ \\
\end{tabular} & & & 2.00 & $-5.6 \mathrm{E}-07$ & & \\
\hline 40 & 136.58 & -2.47 & 2.03 & \begin{tabular}{|l|}
$2.04 \mathrm{E}-07$ \\
\end{tabular} & 2.21 & $1.33 \mathrm{E}-03$ & & & & \\
\hline 30 & 139.73 & -3.25 & 2.00 & $2.94 \mathrm{E}-07$ & & & 1.99 & $-1.34 \mathrm{E}-06$ & & \\
\hline
\end{tabular}

Best fit:

\begin{tabular}{|c|c|c|c|c|c|c|c|c|c|c|c|}
\hline & & & & $3 \times Z$ & N/O - I & & In-S & $1 \times Z$ & N/O - II & $1 \times \mathrm{Zn}-$ & /O - III \\
\hline Time (ms) & Residual & $\chi^{2}$ & $\Delta \mathbf{E}_{0}$ & $\mathbf{R}[\AA ̊ \AA]$ & $\sigma^{2}\left[\AA^{2}\right]$ & $\mathbf{R}[\AA]$ & $\sigma^{2}\left[\AA^{2}\right]$ & $\mathbf{R}[\AA]$ & $\sigma^{2}\left[\AA^{2}\right]$ & $\mathbf{R}[\AA \AA]$ & $\sigma^{2}\left[\AA^{2}\right]$ \\
\hline 600 & 80 & 0.30 & -7.70 & $1.94(1)$ & $1.00 \mathrm{E}-03$ & & & $2.08(1)$ & $1.00 \mathrm{E}-03$ & & \\
\hline
\end{tabular}

$\underline{\text { Curve fitting analysis: }}$

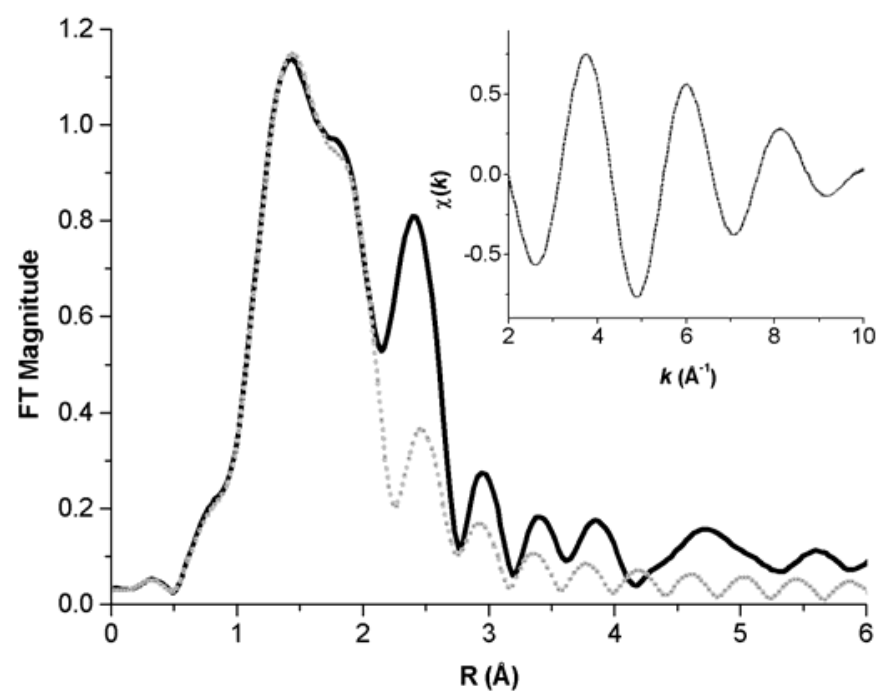




\section{$\underline{700 \mathrm{~ms}}$}

RPA:

\begin{tabular}{|c|c|c|c|c|c|c|c|c|c|c|}
\hline & & & \multicolumn{2}{|c|}{$3 \times \mathrm{Zn}-\mathrm{N} / \mathrm{O}-\mathrm{I}$} & \multicolumn{2}{|c|}{$1 \times \mathrm{Zn}-\mathrm{S}$} & \multicolumn{2}{|c|}{$1 \times \mathrm{Zn}-\mathrm{N} / O-\mathrm{II}$} & \multicolumn{2}{|c|}{ 1x Zn-N/O - III } \\
\hline Residual & $\chi^{2}$ & $\Delta \mathbf{E}_{0}$ & $\mathbf{R}[\AA]$ & $\sigma^{2}\left[\AA^{2}\right]$ & $\mathbf{R}[\AA]$ & $\sigma^{2}\left[\AA^{2}\right]$ & $\mathbf{R}[\AA]$ & $\sigma^{2}\left[\AA^{2}\right]$ & $\mathbf{R}[\AA]$ & $\sigma^{2}\left[\AA^{2}\right]$ \\
\hline 80 & 26.45 & -11.91 & 2.03 & $-7.40 \mathrm{E}-04$ & & & 1.84 & $-9.10 \mathrm{E}-07$ & 1.84 & $-9.10 \mathrm{E}-07$ \\
\hline 70 & 28.82 & -11.27 & 2.04 & $-9.50 \mathrm{E}-04$ & & & 1.85 & $-5.10 \mathrm{E}-07$ & 1.85 & $-5.10 \mathrm{E}-07$ \\
\hline 90 & 33.02 & -11.89 & 2.02 & $-8.60 \mathrm{E}-04$ & & & 1.84 & $-4.70 \mathrm{E}-04$ & 1.84 & $-4.70 \mathrm{E}-04$ \\
\hline 60 & 36.80 & -10.99 & 2.06 & $-1.22 \mathrm{E}-03$ & & & 1.86 & 1.06E-07 & 1.86 & $1.06 \mathrm{E}-07$ \\
\hline 100 & 39.01 & -12.07 & 2.01 & \begin{tabular}{|l|}
$8.95 \mathrm{E}-04$ \\
\end{tabular} & & & 1.83 & $1.06 \mathrm{E}-03$ & 1.83 & $1.06 \mathrm{E}-03$ \\
\hline 50 & 47.93 & -10.74 & 2.06 & $-1.30 \mathrm{E}-03$ & & & 1.87 & 3.84E-07 & 1.87 & $3.84 \mathrm{E}-07$ \\
\hline 40 & 53.45 & -12.03 & 2.07 & $-1.11 \mathrm{E}-03$ & & & 1.87 & $-3.50 \mathrm{E}-07$ & 1.87 & $-3.50 \mathrm{E}-07$ \\
\hline 30 & 55.50 & -15.74 & 2.04 & $-2.50 \mathrm{E}-04$ & & & 1.86 & 3.45E-07 & 1.86 & $3.45 \mathrm{E}-07$ \\
\hline 60 & 79.02 & -14.07 & 1.98 & 5.07E-04 & & & 1.80 & 9.57E-07 & & \\
\hline 40 & 110.66 & -12.62 & 2.02 & \begin{tabular}{|l|}
$-1.59 \mathrm{E}-03$ \\
\end{tabular} & & & 1.85 & $-3.10 \mathrm{E}-07$ & & \\
\hline 70 & 114.98 & -12.33 & 1.99 & $1.46 \mathrm{E}-03$ & & & 1.82 & $-4.70 \mathrm{E}-07$ & & \\
\hline 50 & 126.00 & -11.55 & 2.02 & \begin{tabular}{|l|}
$-2.42 \mathrm{E}-03$ \\
\end{tabular} & & & 1.85 & $4.23 \mathrm{E}-07$ & & \\
\hline 80 & 129.17 & -14.25 & 1.95 & $2.27 \mathrm{E}-07$ & & & 1.89 & $1.70 \mathrm{E}-07$ & & \\
\hline 100 & 132.38 & -12.25 & 1.98 & 7.84E-04 & & & 1.80 & 6.43E-07 & & \\
\hline 80 & 138.19 & -11.72 & 2.00 & $-1.73 \mathrm{E}-03$ & & & 1.82 & $-2.70 \mathrm{E}-07$ & & \\
\hline 90 & 140.70 & -11.81 & 1.99 & $1.49 \mathrm{E}-03$ & & & 1.82 & $9.40 \mathrm{E}-07$ & & \\
\hline 100 & 178.57 & -11.83 & 1.97 & $-1.14 \mathrm{E}-03$ & & & 1.93 & 4.18E-07 & & \\
\hline 90 & 183.92 & -11.45 & 1.98 & $1.55 \mathrm{E}-03$ & & & 1.94 & $-1.50 \mathrm{E}-07$ & & \\
\hline
\end{tabular}

Best fit:

\begin{tabular}{|c|c|c|c|c|c|c|c|c|c|c|c|}
\hline \multirow[b]{2}{*}{ Time (ms) } & \multirow[b]{2}{*}{ Residual } & \multirow[b]{2}{*}{$\chi^{2}$} & \multirow[b]{2}{*}{$\Delta \mathbf{E}_{0}$} & \multicolumn{2}{|c|}{$3 \times \mathrm{Zn}-\mathrm{N} / \mathrm{O}-\mathrm{I}$} & \multicolumn{2}{|c|}{$1 \times \mathrm{Zn}-\mathrm{S}$} & \multicolumn{2}{|c|}{1 x Zn-N/O - II } & \multicolumn{2}{|c|}{ 1x Zn-N/O - III } \\
\hline & & & & $\mathbf{R}[\AA ̊]$ & $\sigma^{2}\left[\AA^{2}\right]$ & $\mathbf{R}[\AA]$ & $\sigma^{2}\left[\AA^{2}\right]$ & $\mathbf{R}[\AA]$ & $\sigma^{2}\left[\AA^{2}\right]$ & $\mathbf{R}[\AA ̊]$ & $\sigma^{2}\left[\AA^{2}\right]$ \\
\hline 700 & 80 & 7.80 & -14.00 & $1.97(1)$ & 2.10E-03 & & & $1.80(1)$ & $1.00 \mathrm{E}-03 \mathrm{~F}$ & & \\
\hline
\end{tabular}

Curve fitting analysis:

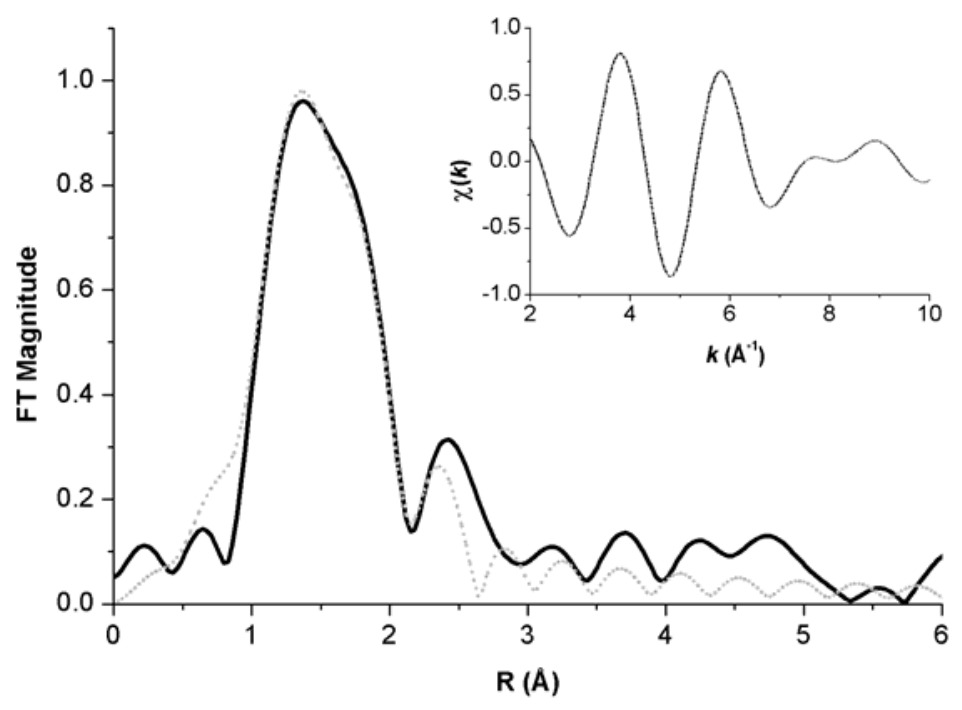


RPA:

$\underline{800 \mathrm{~ms}}$

\begin{tabular}{|c|c|c|c|c|c|c|c|c|c|c|}
\hline & & & \multicolumn{2}{|c|}{$3 \times \mathrm{Zn}-\mathrm{N} / \mathrm{O}-\mathrm{I}$} & \multicolumn{2}{|c|}{$1 \times \mathrm{Zn}-\mathrm{S}$} & \multicolumn{2}{|c|}{$1 \times \mathrm{Zn}-\mathrm{N} / \mathrm{O}$ - II } & \multicolumn{2}{|c|}{$1 \times \mathrm{Zn}-\mathrm{N} / \mathrm{O}-\mathrm{III}$} \\
\hline Residual & $\chi^{2}$ & $\Delta \mathrm{E}_{0}$ & $\mathbf{R}[\mathbf{A}]$ & $\sigma^{2}\left[\AA^{2}\right]$ & $\mathbf{R}[\mathbf{A}]$ & $\sigma^{2}\left[\AA^{2}\right]$ & $\mathbf{R}[\AA]$ & $\sigma^{2}\left[\AA^{2}\right]$ & $\mathbf{R}[\AA]$ & $\sigma^{2}\left[\AA^{2}\right]$ \\
\hline 100 & 24.14 & 7.44 & 2.12 & $2.24 \mathrm{E}-03$ & & & 1.95 & $1.06 \mathrm{E}-06$ & & \\
\hline 80 & 26.86 & 5.93 & 2.04 & \begin{tabular}{|l|}
$3.36 \mathrm{E}-03$ \\
\end{tabular} & & & 2.11 & 3.75E-04 & & \\
\hline 90 & 29.03 & 5.75 & 2.03 & \begin{tabular}{|l|}
$3.95 \mathrm{E}-03$ \\
\end{tabular} & & & 2.11 & $-4.62 \mathrm{E}-07$ & & \\
\hline 80 & 29.53 & 5.17 & 2.09 & $-1.15 \mathrm{E}-03$ & & & 1.96 & \begin{tabular}{|l|}
$8.5 \mathrm{E}-07$ \\
\end{tabular} & & \\
\hline 70 & 29.73 & 5.49 & 2.04 & $1.33 \mathrm{E}-03$ & & & 2.15 & $-2.18 \mathrm{E}-06$ & & \\
\hline 40 & 31.88 & -0.54 & 2.05 & \begin{tabular}{|l|}
$8.37 \mathrm{E}-04$ \\
\end{tabular} & 2.25 & $5.72 \mathrm{E}-03$ & 1.97 & $-4.6 \mathrm{E}-07$ & & \\
\hline 60 & 32.77 & 4.34 & 2.05 & -1.54E-03 & & & 2.07 & $2.01 \mathrm{E}-03$ & & \\
\hline 50 & 32.80 & 2.80 & 2.04 & -7.39E-04 & & & 2.01 & $-3.8 \mathrm{E}-07$ & & \\
\hline 90 & 37.75 & 8.24 & 2.13 & $5.05 \mathrm{E}-03$ & & & 2.02 & $1.21 \mathrm{E}-06$ & & \\
\hline 50 & 41.48 & 4.69 & 2.08 & \begin{tabular}{|l|}
$-5.48 \mathrm{E}-04$ \\
\end{tabular} & & & 2.02 & $-8.99 \mathrm{E}-08$ & & \\
\hline 100 & 43.32 & 4.28 & 2.08 & $2.98 \mathrm{E}-04$ & & & 1.91 & $2.58 \mathrm{E}-08$ & & \\
\hline 50 & 45.31 & 3.53 & 2.06 & $9.20 \mathrm{E}-07$ & 2.30 & $7.66 \mathrm{E}-03$ & & & & \\
\hline
\end{tabular}

Best fit:

\begin{tabular}{|c|c|c|c|c|c|c|c|c|c|c|c|}
\hline & & & & $3 \times Z$ & N/O - I & & $n-S$ & $1 \times Z$ & N/O - II & $1 \times \mathrm{Zn}-$ & O - III \\
\hline Time (ms) & Residual & $\chi^{2}$ & $\Delta \mathrm{E}_{0}$ & $\mathbf{R}[\AA]$ & $\sigma^{2}\left[\AA^{2}\right]$ & $\mathbf{R}[\AA]$ & $\sigma^{2}\left[\AA^{2}\right]$ & $\mathbf{R}[\AA \mathbf{A}]$ & $\sigma^{2}\left[\AA^{2}\right]$ & $\mathbf{R}[\AA ̊]$ & $\sigma^{2}\left[\AA^{2}\right]$ \\
\hline 800 & 80 & 0.72 & -7.00 & $2.01(1)$ & $2.00 \mathrm{E}-03$ & & & $1.83(2)$ & $2.00 \mathrm{E}-03$ & & \\
\hline
\end{tabular}

Curve fitting analysis:

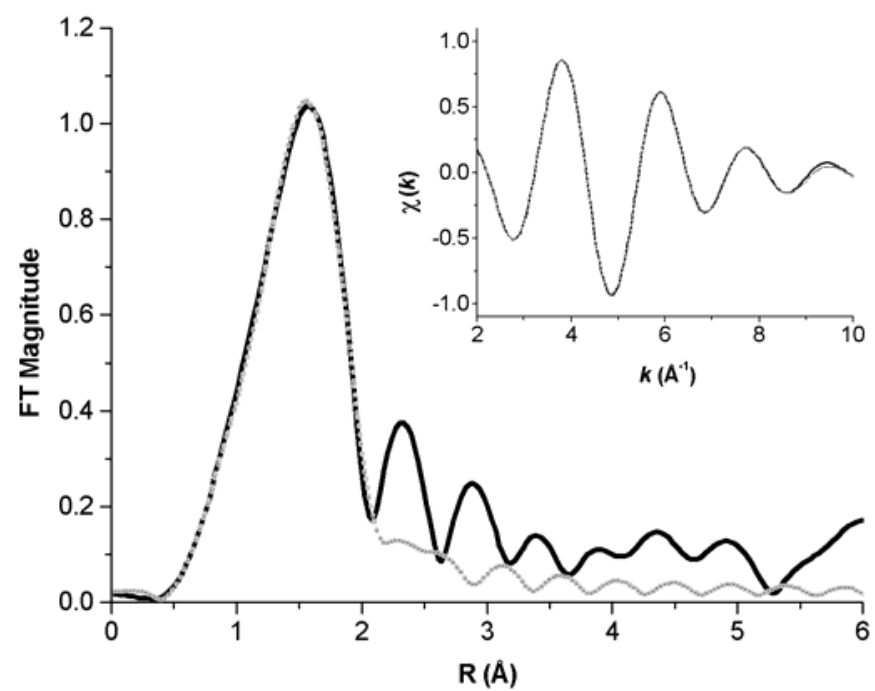


RPA:

$\underline{900 \mathrm{~ms}}$

\begin{tabular}{|c|c|c|c|c|c|c|c|c|c|c|}
\hline \multirow[b]{2}{*}{ Residual } & \multirow[b]{2}{*}{$\chi^{2}$} & \multirow[b]{2}{*}{$\Delta \mathrm{E}_{0}$} & \multicolumn{2}{|c|}{$3 \times \mathrm{Zn}-\mathrm{N} / \mathrm{O}-\mathrm{I}$} & \multicolumn{2}{|c|}{$1 \times \mathrm{Zn}-\mathrm{S}$} & \multicolumn{2}{|c|}{$1 \times \mathrm{Zn}-\mathrm{N} / \mathrm{O}$ - II } & \multicolumn{2}{|c|}{ 1x Zn-N/O - III } \\
\hline & & & $\mathbf{R}[\AA]$ & $\sigma^{2}\left[\AA^{2}\right]$ & $\mathbf{R}[\AA]$ & $\sigma^{2}\left[\AA^{2}\right]$ & $\mathbf{R}[\AA]$ & $\sigma^{2}\left[\AA^{2}\right]$ & $\mathbf{R}[\AA]$ & $\sigma^{2}\left[\AA^{2}\right]$ \\
\hline 70 & 6.84 & -5.40 & 1.90 & $1.10 \mathrm{E}-07$ & & & 2.06 & $6.93 \mathrm{E}-03$ & & \\
\hline 90 & 7.36 & -2.21 & 1.93 & \begin{tabular}{|l|}
$4.47 \mathrm{E}-04$ \\
\end{tabular} & 2.35 & $3.03 \mathrm{E}-05$ & & & & \\
\hline 100 & 7.90 & -2.03 & 1.93 & 6.75E-04 & 2.35 & $-6.50 \mathrm{E}-05$ & & & & \\
\hline 80 & 7.94 & -4.56 & 1.90 & $-1.60 \mathrm{E}-07$ & & & 2.05 & $2.19 \mathrm{E}-03$ & & \\
\hline 90 & 8.98 & -1.51 & 1.91 & \begin{tabular}{|l|}
$-3.20 \mathrm{E}-07$ \\
\end{tabular} & & & 2.08 & $6.24 \mathrm{E}-03$ & 2.08 & $6.24 \mathrm{E}-03$ \\
\hline 90 & 9.25 & -4.02 & 1.90 & $-1.10 \mathrm{E}-06$ & & & 2.05 & $-1.90 \mathrm{E}-06$ & & \\
\hline 100 & 9.95 & -0.94 & 1.91 & -2.50E-06 & & & 2.08 & $4.51 \mathrm{E}-03$ & 2.08 & $4.51 \mathrm{E}-03$ \\
\hline 100 & 11.57 & -4.04 & 1.90 & \begin{tabular}{|l|}
$-6.10 \mathrm{E}-04$ \\
\end{tabular} & & & 2.04 & $3.40 \mathrm{E}-06$ & & \\
\hline 90 & 14.67 & -2.14 & 1.92 & \begin{tabular}{|l|}
$6.29 \mathrm{E}-03$ \\
\end{tabular} & 2.39 & $1.24 \mathrm{E}-06$ & 1.93 & $-5.40 \mathrm{E}-04$ & & \\
\hline 20 & 37.83 & -2.55 & 1.89 & \begin{tabular}{|l|}
$-9.70 \mathrm{E}-07$ \\
\end{tabular} & & & 1.89 & $-8.70 \mathrm{E}-06$ & & \\
\hline 10 & 50.22 & -0.83 & 1.87 & $-2.40 \mathrm{E}-06$ & & & 1.87 & 2.31E-06 & 1.87 & $2.31 \mathrm{E}-06$ \\
\hline 10 & 53.03 & 0.03 & 1.88 & $1.36 \mathrm{E}-07$ & & & 1.87 & $1.48 \mathrm{E}-07$ & & \\
\hline 10 & 54.15 & 0.49 & 1.89 & \begin{tabular}{|l|}
$2.35 \mathrm{E}-08$ \\
\end{tabular} & & & 2.00 & \begin{tabular}{|l|}
$9.26 \mathrm{E}-07$ \\
\end{tabular} & & \\
\hline
\end{tabular}

Best fit:

\begin{tabular}{|c|c|c|c|c|c|c|c|c|c|c|c|}
\hline \multirow[b]{2}{*}{ Time (ms) } & \multirow[b]{2}{*}{ Residual } & \multirow[b]{2}{*}{$\chi^{2}$} & \multirow[b]{2}{*}{$\Delta \mathbf{E}_{0}$} & \multicolumn{2}{|c|}{$3 \times \mathrm{Zn}-\mathrm{N} / \mathrm{O}-\mathrm{I}$} & \multicolumn{2}{|c|}{$1 \times \mathrm{Zn}-\mathrm{S}$} & \multicolumn{2}{|c|}{$1 \times \mathrm{Zn}-\mathrm{N} / \mathrm{O}$ - II } & \multicolumn{2}{|c|}{ 1x Zn-N/O - III } \\
\hline & & & & $\mathbf{R}[\AA]$ & $\sigma^{2}\left[\AA^{2}\right]$ & $\mathbf{R}[\AA]$ & $\sigma^{2}\left[\AA^{2}\right]$ & $\mathbf{R}[\AA]$ & $\sigma^{2}\left[\AA^{2}\right]$ & $\mathbf{R}[\AA]$ & $\sigma^{2}\left[\AA^{2}\right]$ \\
\hline 900 & 70 & 1.47 & -4.40 & $1.93(1)$ & 4.90E-03 & & & $1.93(1)$ & 4.90E-03 & & \\
\hline
\end{tabular}

Curve fitting analysis:

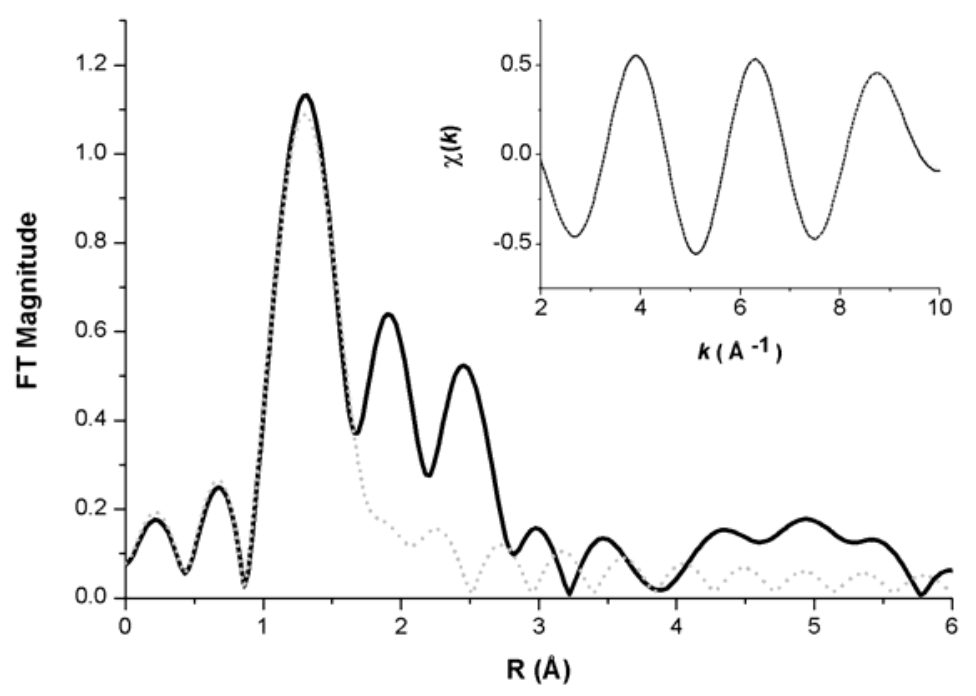


RPA:

\section{$1000 \mathrm{~ms}$}

\begin{tabular}{|c|c|c|c|c|c|c|c|c|c|c|}
\hline \multirow[b]{2}{*}{ Residual } & \multirow[b]{2}{*}{$\chi^{2}$} & \multirow[b]{2}{*}{$\Delta \mathbf{E}_{0}$} & \multicolumn{2}{|c|}{$3 \times \mathrm{Zn}-\mathrm{N} / \mathrm{O}-\mathrm{I}$} & \multicolumn{2}{|c|}{$1 \times \mathrm{Zn}-\mathrm{S}$} & \multicolumn{2}{|c|}{1 x Zn-N/O - II } & \multicolumn{2}{|c|}{$1 \times \mathrm{Zn}-\mathrm{N} / \mathrm{O}-$ III } \\
\hline & & & $\mathbf{R}[\AA]$ & $\sigma^{2}\left[\AA^{2}\right]$ & $\mathbf{R}[\AA ̊]$ & $\sigma^{2}\left[\AA^{2}\right]$ & $\mathbf{R}[\AA]$ & $\sigma^{2}\left[\AA^{2}\right]$ & $\mathbf{R}[\AA ̊]$ & $\sigma^{2}\left[\AA^{2}\right]$ \\
\hline 100 & 19.39 & -1.13 & 2.01 & 4.77E-03 & & & 2.00 & 4.77E-03 & 2.58 & $-8.12 \mathrm{E}-07$ \\
\hline 90 & 22.23 & -3.12 & 2.00 & 3.53E-03 & & & 1.99 & 3.53E-03 & 2.56 & $-1.05 E-06$ \\
\hline 70 & 22.58 & -6.04 & 1.98 & $-8.82 E-04$ & & & 1.97 & $-8.82 E-04$ & 2.56 & $-2.95 \mathrm{E}-06$ \\
\hline 80 & 23.90 & -4.13 & 1.99 & $2.30 \mathrm{E}-03$ & & & 1.98 & 2.30E-03 & 2.57 & $-1.24 \mathrm{E}-06$ \\
\hline 70 & 30.59 & -4.60 & 2.00 & $-7.32 \mathrm{E}-04$ & & & 1.94 & 7.43E-08 & & \\
\hline 70 & 71.66 & -4.60 & 2.00 & $-2.05 E-07$ & 2.19 & 8.19E-03 & & & & \\
\hline 50 & 103.59 & -3.54 & 2.00 & $-1.86 \mathrm{E}-07$ & & & 1.96 & $-1.79 \mathrm{E}-08$ & & \\
\hline 60 & 131.89 & -4.78 & 2.01 & $-1.91 \mathrm{E}-07$ & 2.17 & 4.37E-03 & & & & \\
\hline 40 & 172.38 & -4.28 & 2.02 & $-5.16 \mathrm{E}-07$ & 2.14 & -7.83E-07 & 1.98 & $-1.15 E-06$ & & \\
\hline 50 & 174.13 & -6.11 & 2.01 & $-7.73 E-07$ & 2.15 & 3.23E-06 & & & & \\
\hline 30 & 175.59 & -6.17 & 2.02 & 7.46E-06 & 2.14 & 3.07E-07 & 1.95 & $-6.00 \mathrm{E}-08$ & 1.95 & $-6.00 \mathrm{E}-08$ \\
\hline 30 & 204.02 & -6.87 & 2.01 & 2.06E-07 & & & 1.94 & $-6.11 E-07$ & 1.94 & $-6.11 E-07$ \\
\hline 40 & 224.65 & -2.18 & 2.01 & $-1.11 \mathrm{E}-09$ & & & 2.00 & $-1.07 \mathrm{E}-06$ & & \\
\hline 30 & 270.87 & -3.51 & 2.03 & $-1.21 \mathrm{E}-06$ & 2.14 & 1.00E-06 & 1.97 & 9.34E-07 & & \\
\hline 40 & 290.04 & -3.69 & 2.03 & $2.00 \mathrm{E}-06$ & 2.15 & $1.45 \mathrm{E}-06$ & & & & \\
\hline 30 & 294.63 & -2.78 & 2.01 & -9.10E-09 & & & 1.98 & $-1.22 \mathrm{E}-06$ & & \\
\hline 20 & 323.33 & -4.12 & 1.99 & 4.63E-08 & & & 1.97 & $-9.31 E-07$ & 1.97 & $-9.31 E-07$ \\
\hline 50 & 336.12 & -1.81 & 1.66 & 9.07E-03 & 2.24 & 3.17E-03 & 1.97 & $-1.25 \mathrm{E}-07$ & 1.97 & $-1.25 \mathrm{E}-07$ \\
\hline
\end{tabular}

Best fit:

\begin{tabular}{|c|c|c|c|c|c|c|c|c|c|c|c|}
\hline \multirow[b]{2}{*}{ Time (ms) } & \multirow[b]{2}{*}{ Residual } & \multirow[b]{2}{*}{$\chi^{2}$} & \multirow[b]{2}{*}{$\Delta \mathbf{E}_{0}$} & \multicolumn{2}{|c|}{$3 \times \mathrm{Zn}-\mathrm{N} / \mathrm{O}-\mathrm{I}$} & \multicolumn{2}{|c|}{$1 \times \mathrm{Zn}-\mathrm{S}$} & \multicolumn{2}{|c|}{$1 \times \mathrm{Zn}-\mathrm{N} / \mathrm{O}$ - II } & \multicolumn{2}{|c|}{ 1x Zn-N/O - III } \\
\hline & & & & $\mathbf{R}[\mathbf{A}]$ & $\sigma^{2}\left[\AA^{2}\right]$ & $\mathbf{R}[\AA]$ & $\sigma^{2}\left[\AA^{2}\right]$ & 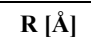 & $\sigma^{2}\left[\AA^{2}\right]$ & $\mathbf{R}[\mathbf{A}]$ & $\sigma^{2}\left[\AA^{2}\right]$ \\
\hline 1000 & 80 & 0.20 & 0.70 & $2.01(1)$ & 2.00E-03 & & & 1.99(1) & 2.00E-03 & $2.19(2)$ & 1.00E-03 \\
\hline
\end{tabular}

Curve fitting analysis:

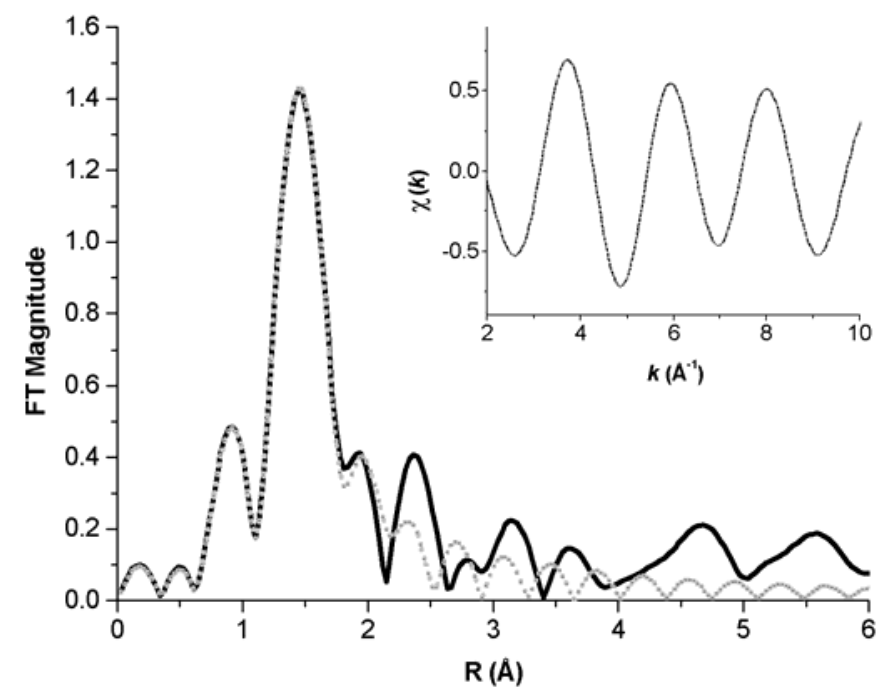




\section{$\underline{1100 \mathrm{~ms}}$}

\section{RPA:}

\begin{tabular}{|c|c|c|c|c|c|c|c|c|c|c|}
\hline \multirow[b]{2}{*}{ Residual } & \multirow[b]{2}{*}{$\chi^{2}$} & \multirow[b]{2}{*}{$\Delta \mathbf{E}_{0}$} & \multicolumn{2}{|c|}{$3 \times \mathrm{Zn}-\mathrm{N} / \mathrm{O}-\mathrm{I}$} & \multicolumn{2}{|c|}{$1 \times \mathrm{Zn}-\mathrm{S}$} & \multicolumn{2}{|c|}{$1 \times \mathrm{Zn}-\mathrm{N} / \mathrm{O}-\mathrm{II}$} & \multicolumn{2}{|c|}{ 1x Zn-N/O-III } \\
\hline & & & 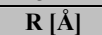 & $\sigma^{2}\left[\AA^{2}\right]$ & $\mathbf{R}[\AA]$ & $\sigma^{2}\left[\AA^{2}\right]$ & $\mathbf{R}[\AA]$ & $\sigma^{2}\left[\AA^{2}\right]$ & $\mathbf{R}[\AA]$ & $\sigma^{2}\left[\AA^{2}\right]$ \\
\hline 70 & 5.3246 & -3.4506 & 1.96777 & $1.94 \mathrm{E}-07$ & & & 2.01261 & 0.00556 & & \\
\hline 80 & 5.63332 & -1.91701 & 1.96845 & $-3.20 \mathrm{E}-05$ & & & 2.04538 & 0.00284 & & \\
\hline 90 & 6.00551 & -1.84033 & 1.9977 & $4.28 \mathrm{E}-03$ & & & 1.9517 & $-2.2 \mathrm{E}-05$ & & \\
\hline 100 & 6.74359 & -0.58499 & 2.02036 & \begin{tabular}{|l|}
$3.77 \mathrm{E}-03$ \\
\end{tabular} & & & 1.93283 & -0.00009 & & \\
\hline 50 & 7.44432 & -6.78796 & 1.97512 & $-7.70 \mathrm{E}-06$ & & & 1.91099 & $7.8 \mathrm{E}-08$ & & \\
\hline 80 & 14.4574 & -2.23312 & 1.95791 & $2.37 \mathrm{E}-04$ & & & 2.0517 & 0.00679 & 2.0517 & 0.00679 \\
\hline 30 & 29.8882 & -8.95441 & 1.97655 & $-4.90 \mathrm{E}-08$ & & & 1.91033 & $1.5 \mathrm{E}-07$ & & \\
\hline
\end{tabular}

Best fit:

\begin{tabular}{|c|c|c|c|c|c|c|c|c|c|c|c|}
\hline \multirow[b]{2}{*}{ Time (ms) } & \multirow[b]{2}{*}{ Residual } & \multirow[b]{2}{*}{$\chi^{2}$} & \multirow[b]{2}{*}{$\Delta \mathbf{E}_{0}$} & \multicolumn{2}{|c|}{$3 \times \mathrm{Zn}-\mathrm{N} / \mathrm{O}-\mathrm{I}$} & \multicolumn{2}{|c|}{$1 \times \mathrm{Zn}-\mathrm{S}$} & \multicolumn{2}{|c|}{$1 \times \mathrm{Zn}-\mathrm{N} / \mathrm{O}$ - II } & \multicolumn{2}{|c|}{ 1x Zn-N/O - III } \\
\hline & & & & $\mathbf{R}[\AA]$ & $\sigma^{2}\left[\AA^{2}\right]$ & $\mathbf{R}[\AA]$ & $\sigma^{2}\left[\AA^{2}\right]$ & $\mathbf{R}[\AA]$ & $\sigma^{2}\left[\AA^{2}\right]$ & $\mathbf{R}[\AA]$ & $\sigma^{2}\left[\AA^{2}\right]$ \\
\hline 1100 & 60 & 4.31 & -6.86 & $1.95(7)$ & 2.00E-03 & & & $2.06(4)$ & $2.00 \mathrm{E}-03$ & $1.95(7)$ & 2.00E-03 \\
\hline
\end{tabular}

Curve fitting analysis:

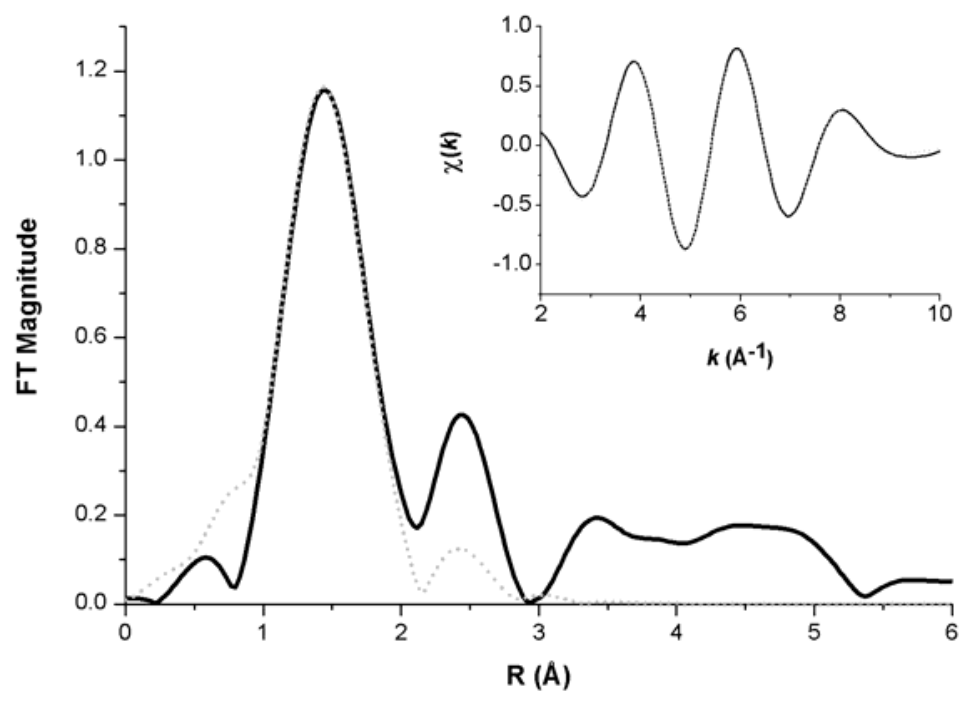




\section{$1200 \mathrm{~ms}$}

RPA:

\begin{tabular}{|c|c|c|c|c|c|c|c|c|c|c|}
\hline \multirow[b]{2}{*}{ Residual } & \multirow[b]{2}{*}{$\chi^{2}$} & \multirow[b]{2}{*}{$\Delta \mathbf{E}_{0}$} & \multicolumn{2}{|c|}{$3 \times \mathrm{Zn}-\mathrm{N} / \mathrm{O}-\mathrm{I}$} & \multicolumn{2}{|c|}{$1 \times \mathrm{Zn}-\mathrm{S}$} & \multicolumn{2}{|c|}{$1 \times \mathrm{Zn}-\mathrm{N} / \mathrm{O}$ - II } & \multicolumn{2}{|c|}{ 1x Zn-N/O - III } \\
\hline & & & $\mathbf{R}[\AA]$ & $\sigma^{2}\left[\AA^{2}\right]$ & $\mathbf{R}[\AA]$ & $\sigma^{2}\left[\AA^{2}\right]$ & $\mathbf{R}[\AA]$ & $\sigma^{2}\left[\AA^{2}\right]$ & $\mathbf{R}[\AA]$ & $\sigma^{2}\left[\AA^{2}\right]$ \\
\hline 70 & 1.06391 & -0.58316 & 2.04715 & $-4.00 \mathrm{E}-07$ & & & 2.00702 & 0.00077 & & \\
\hline 80 & 1.45826 & 0.6965 & 2.03971 & $1.10 \mathrm{E}-03$ & & & 2.04151 & $-4.2 \mathrm{E}-06$ & & \\
\hline 70 & 1.57734 & -6.76658 & 1.99975 & $3.34 \mathrm{E}-07$ & 2.26228 & $-1.03 E-03$ & & & & \\
\hline 90 & 1.65509 & 0.57381 & 2.05688 & $-5.90 \mathrm{E}-08$ & & & 1.97415 & 0.00391 & & \\
\hline 80 & 1.74955 & -4.24096 & 2.01386 & $9.07 \mathrm{E}-05$ & 2.25814 & $3.51 \mathrm{E}-03$ & & & & \\
\hline 100 & 1.80043 & 1.28082 & 2.06337 & $2.47 \mathrm{E}-07$ & & & 1.95945 & 0.00368 & & \\
\hline 90 & 2.13496 & -3.9826 & 2.01246 & $1.07 \mathrm{E}-03$ & 2.25328 & $-2.87 \mathrm{E}-03$ & & & & \\
\hline 60 & 2.44167 & -2.71007 & 2.07054 & $-5.1 \mathrm{E}-08$ & & & 1.96822 & 0.00085 & 3.88342 & 0.00085 \\
\hline 90 & 2.53154 & -0.52536 & 2.04633 & $3.5 \mathrm{E}-09$ & & & 1.985 & 0.01437 & 3.9002 & 0.01437 \\
\hline 100 & 2.59291 & -4.15601 & 2.0073 & $2.04 \mathrm{E}-03$ & 2.22797 & $1.92 \mathrm{E}-03$ & & & & \\
\hline 100 & 2.98042 & -1.48482 & 2.05221 & $-2.30 \mathrm{E}-07$ & & & 1.93913 & 0.00932 & 3.85433 & 0.00932 \\
\hline 70 & 4.28763 & -1.39954 & 2.07685 & $-1.00 \mathrm{E}-06$ & & & 1.96353 & 0.00216 & 3.87873 & 0.00216 \\
\hline 80 & 4.54308 & -0.71246 & 2.07147 & $2.58 \mathrm{E}-07$ & & & 1.95976 & 0.00505 & 3.87496 & 0.00505 \\
\hline 60 & 15.7908 & 3.27609 & 2.09789 & $2.87 \mathrm{E}-04$ & & & 2.00413 & $4.9 \mathrm{E}-08$ & & \\
\hline 30 & 29.4541 & -7.96027 & 2.02413 & $-6.70 \mathrm{E}-07$ & 2.1818 & $-7.10 \mathrm{E}-07$ & & & & \\
\hline 50 & 32.4143 & 5.15827 & 2.17536 & $8.86 \mathrm{E}-03$ & & & 2.03399 & $-2.6 \mathrm{E}-08$ & 3.94919 & $-2.6 \mathrm{E}-08$ \\
\hline
\end{tabular}

Best fit:

\begin{tabular}{|c|c|c|c|c|c|c|c|c|c|c|c|}
\hline \multirow[b]{2}{*}{ Time (ms) } & \multirow[b]{2}{*}{ Residual } & \multirow[b]{2}{*}{$\chi^{2}$} & \multirow[b]{2}{*}{$\Delta \mathbf{E}_{0}$} & \multicolumn{2}{|c|}{$3 \times \mathrm{Zn}-\mathrm{N} / \mathrm{O}-\mathrm{I}$} & \multicolumn{2}{|c|}{$1 \times \mathrm{Zn}-\mathrm{S}$} & \multicolumn{2}{|c|}{$1 \times \mathrm{Zn}-\mathrm{N} / \mathrm{O}-\mathrm{II}$} & \multicolumn{2}{|c|}{ 1x Zn-N/O - III } \\
\hline & & & & $\mathbf{R}[\AA]$ & $\sigma^{2}\left[\AA^{2}\right]$ & $\mathbf{R}[\AA]$ & $\sigma^{2}\left[\AA^{2}\right]$ & $\mathbf{R}[\AA]$ & $\sigma^{2}\left[\AA^{2}\right]$ & $\mathbf{R}[\AA \AA]$ & $\sigma^{2}\left[\AA^{2}\right]$ \\
\hline 1200 & 60 & 2.21 & -2.71 & $2.03(1)$ & $1.70 \mathrm{E}-03$ & & & $2.02(1)$ & 1.70E-03 & $2.02(1)$ & $1.70 \mathrm{E}-03$ \\
\hline
\end{tabular}

Curve fitting analysis:

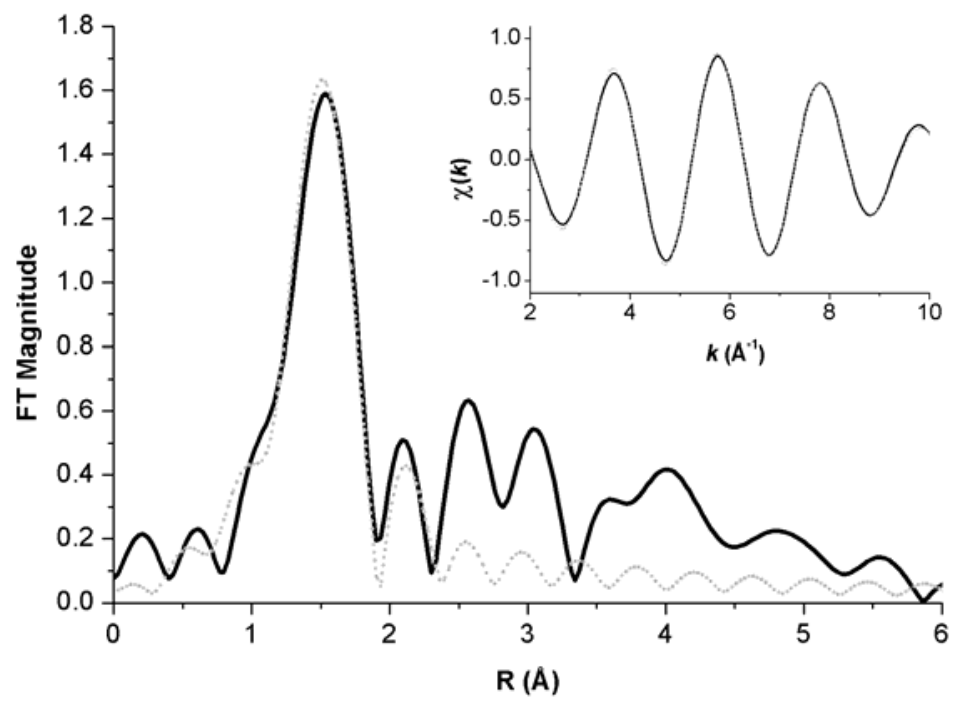




\section{$1400 \mathrm{~ms}$}

RPA:

\begin{tabular}{|c|c|c|c|c|c|c|c|c|c|c|}
\hline \multirow[b]{2}{*}{ Residual } & \multirow[b]{2}{*}{$\chi^{2}$} & \multirow[b]{2}{*}{$\Delta \mathbf{E}_{0}$} & \multicolumn{2}{|c|}{$3 \times \mathrm{Zn}-\mathrm{N} / \mathrm{O}$ - I } & \multicolumn{2}{|c|}{$1 \times \mathrm{Zn}-\mathrm{S}$} & \multicolumn{2}{|c|}{$1 \times \mathrm{Zn}-\mathrm{N} / \mathrm{O}-\mathrm{II}$} & \multicolumn{2}{|c|}{ 1x Zn-N/O - III } \\
\hline & & & $\mathbf{R}[\AA]$ & $\sigma^{2}\left[\AA^{2}\right]$ & $\mathbf{R}[\mathbf{A}]$ & $\sigma^{2}\left[\AA^{2}\right]$ & $\mathbf{R}[\AA]$ & $\sigma^{2}\left[\AA^{2}\right]$ & $\mathbf{R}[\AA]$ & $\sigma^{2}\left[\AA^{2}\right]$ \\
\hline 70 & 22.2945 & 5.77858 & 2.00467 & 0.00443 & & & 2.11589 & $2.3 \mathrm{E}-06$ & 2.11589 & $2.3 \mathrm{E}-06$ \\
\hline 80 & 28.7623 & 5.79595 & 2.00119 & 0.00459 & & & 2.11288 & $3.6 \mathrm{E}-06$ & 2.11288 & $3.57 \mathrm{E}-06$ \\
\hline 70 & 32.2645 & 1.97811 & 2.02211 & 0.0038 & 2.3316 & 1.21E-03 & & & & \\
\hline 90 & 35.7798 & 5.84272 & 1.99804 & 0.00451 & & & 2.11207 & 1.3E-06 & 2.11207 & $1.3 \mathrm{E}-06$ \\
\hline 100 & 43.4297 & 5.75878 & 1.99457 & 0.0046 & & & 2.10913 & $8.5 \mathrm{E}-07$ & 2.10913 & $8.5 \mathrm{E}-07$ \\
\hline 80 & 45.4969 & 2.02397 & 2.01921 & 0.00399 & 2.32934 & $1.43 \mathrm{E}-03$ & & & & \\
\hline 90 & 61.6667 & 2.05293 & 2.01683 & 0.00413 & 2.32747 & $1.58 \mathrm{E}-03$ & & & & \\
\hline 100 & 79.98 & 2.06199 & 2.01473 & 0.00423 & 2.32579 & $1.69 \mathrm{E}-03$ & & & & \\
\hline 60 & 113.104 & -2.22565 & 2.04059 & $1 \mathrm{E}-04$ & & & 1.96774 & $2.8 \mathrm{E}-06$ & & \\
\hline 70 & 121.603 & -1.78241 & 2.02334 & 0.00133 & & & 2.01175 & 0.00043 & & \\
\hline 80 & 122.41 & -1.34802 & 2.0508 & 0.00102 & & & 1.95316 & $-2.6 \mathrm{E}-05$ & & \\
\hline 100 & 126.644 & 7.87475 & 2.11973 & 0.00201 & & & 2.09444 & $-4.1 \mathrm{E}-06$ & & \\
\hline 90 & 137.356 & -0.92409 & 2.05471 & 0.00153 & & & 1.95187 & $-1.3 \mathrm{E}-06$ & & \\
\hline 100 & 158.492 & -0.70037 & 2.05196 & 0.00222 & & & 1.95796 & 0.00075 & & \\
\hline 60 & 322.207 & -2.27958 & 2.01756 & $3.3 \mathrm{E}-06$ & & & 1.94266 & $5.1 \mathrm{E}-06$ & & \\
\hline
\end{tabular}

Best fit:

\begin{tabular}{|c|c|c|c|c|c|c|c|c|c|c|c|}
\hline \multirow[b]{2}{*}{ Time (ms) } & \multirow[b]{2}{*}{ Residual } & \multirow[b]{2}{*}{$\chi^{2}$} & \multirow[b]{2}{*}{$\Delta \mathbf{E}_{0}$} & \multicolumn{2}{|c|}{$3 \times \mathrm{Zn}-\mathrm{N} / \mathrm{O}-\mathrm{I}$} & \multicolumn{2}{|c|}{$1 \times \mathrm{Zn}-\mathrm{S}$} & \multicolumn{2}{|c|}{$1 \times \mathrm{Zn}-\mathrm{N} / \mathrm{O}$ - II } & \multicolumn{2}{|c|}{ 1x Zn-N/O - III } \\
\hline & & & & $\mathbf{R}[\AA ̊]$ & $\sigma^{2}\left[\AA^{2}\right]$ & $\mathbf{R}[\AA]$ & $\sigma^{2}\left[\AA^{2}\right]$ & $\mathbf{R}[\AA]$ & $\sigma^{2}\left[\AA^{2}\right]$ & $\mathbf{R}[\AA]$ & $\overline{\sigma^{2}\left[\AA^{2}\right]}$ \\
\hline 1400 & 60 & 28.75 & 7.77 & $2.13(2)$ & $7.00 \mathrm{E}-04$ & & & $1.96(5)$ & $5.00 \mathrm{E}-03 \mathrm{~F}$ & & \\
\hline
\end{tabular}

Curve fitting analysis:

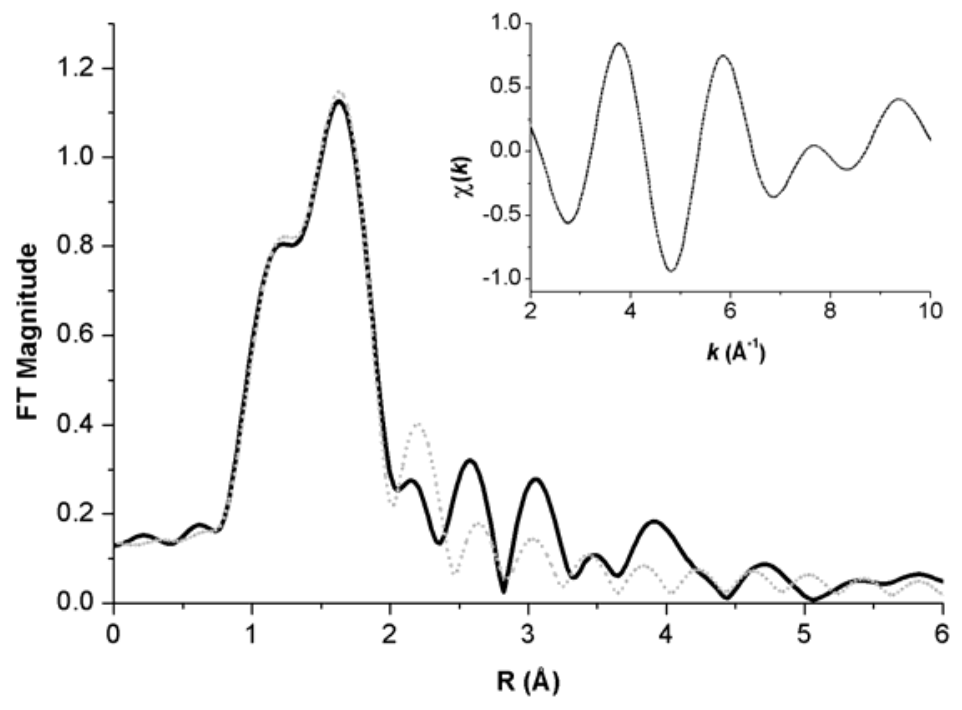




\section{Pro-MMP-9}

Best fit:

\begin{tabular}{|c|c|c|c|c|c|c|c|c|c|c|c|}
\hline \multirow[b]{2}{*}{ Time (ms) } & \multirow[b]{2}{*}{ Residual } & \multirow[b]{2}{*}{$\chi^{2}$} & \multirow[b]{2}{*}{$\Delta \mathbf{E}_{0}$} & \multicolumn{2}{|c|}{$3 \times \mathrm{Zn}-\mathrm{N} / \mathrm{O}$ - I } & \multicolumn{2}{|c|}{$1 \times \mathrm{Zn}-\mathrm{S}$} & \multicolumn{2}{|c|}{$1 \times \mathrm{Zn}-\mathrm{N} / \mathrm{O}-\mathrm{II}$} & \multicolumn{2}{|c|}{ 1x Zn-N/O - III } \\
\hline & & & & $\mathbf{R}[\AA]$ & $\sigma^{2}\left[\AA^{2}\right]$ & $\mathbf{R}[\AA]$ & $\sigma^{2}\left[\AA^{2}\right]$ & $\mathbf{R}[\AA]$ & $\sigma^{2}\left[\AA^{2}\right]$ & $\mathbf{R}[\AA]$ & $\sigma^{2}\left[\AA^{2}\right]$ \\
\hline Pro & 100 & 6.21 & -5.05 & $1.93(1)$ & 4.40E-03 & $2.26(4)$ & 4.00E-04 & & & & \\
\hline
\end{tabular}

$\underline{\text { Curve fitting analysis: }}$

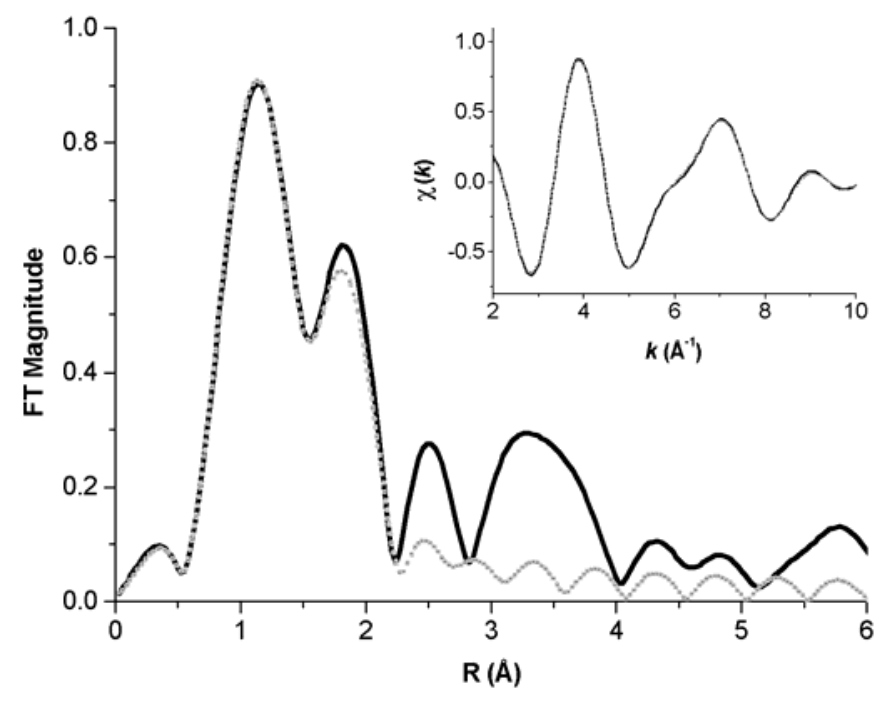




\section{Active MMP-9}

\section{Best fit:}

\begin{tabular}{|c|c|c|c|c|c|c|c|c|c|c|c|}
\hline \multirow[b]{2}{*}{ Time (ms) } & \multirow[b]{2}{*}{ Residual } & \multirow[b]{2}{*}{$\chi^{2}$} & \multirow[b]{2}{*}{$\Delta \mathbf{E}_{0}$} & \multicolumn{2}{|c|}{$3 \times \mathrm{Zn}-\mathrm{N} / \mathrm{O}-\mathrm{I}$} & \multicolumn{2}{|c|}{$1 \times \mathrm{Zn}-\mathrm{S}$} & \multicolumn{2}{|c|}{$1 \times \mathrm{Zn}-\mathrm{N} / \mathrm{O}$ - II } & \multicolumn{2}{|c|}{ 1x Zn-N/O - III } \\
\hline & & & & $\mathbf{R}[\AA]$ & $\sigma^{2}\left[\AA^{2}\right]$ & $\mathbf{R}[\AA ̊]$ & $\sigma^{2}\left[\AA^{2}\right]$ & $\mathbf{R}[\AA]$ & $\sigma^{2}\left[\AA^{2}\right]$ & $\mathbf{R}[\AA ̊]$ & $\sigma^{2}\left[\AA^{2}\right]$ \\
\hline Active & 100 & 0.40 & -2.30 & $2.02(1)$ & $1.00 \mathrm{E}-03$ & & & $1.89(1)$ & $1.00 \mathrm{E}-03$ & & \\
\hline
\end{tabular}

\section{Curve fitting analysis:}

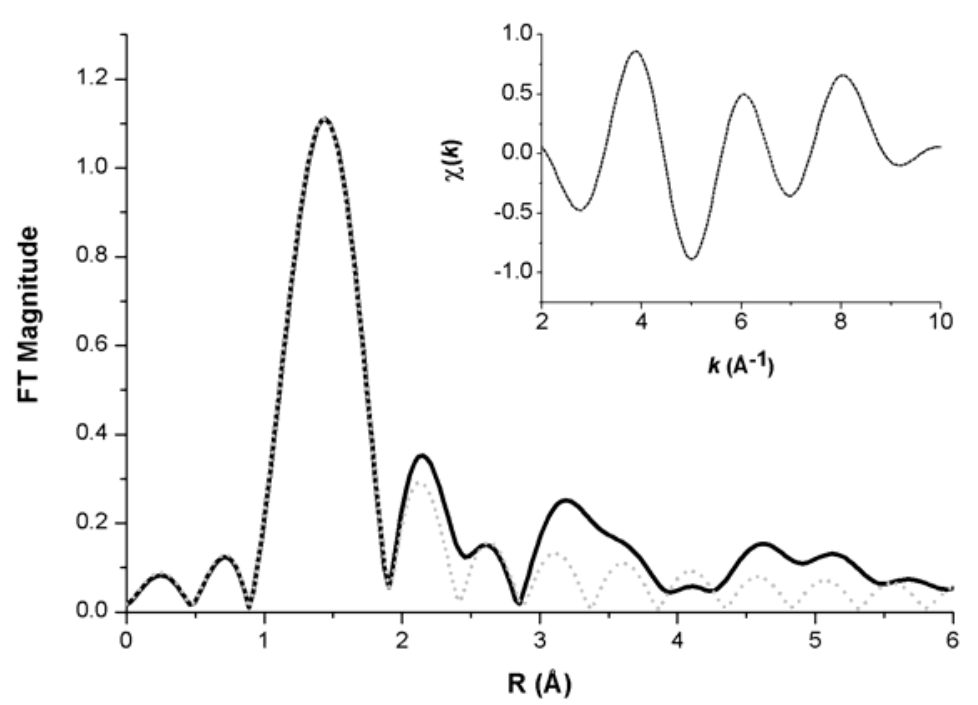




\section{Refrerences}

1. Frenkel, A. I.; Kleifeld, O.; Wasserman, S. R.; Sagi, I., J. Chem. Phys. 2002, 116, (21), 9449-9456.

2. $\quad$ Gabb, H. A.; Jackson, R. M.; Sternberg, M. J., J Mol Biol 1997, 272, (1), 106-

120.

3. Jackson, R. M.; Gabb, H. A.; Sternberg, M. J., J Mol Biol 1998, 276, (1), 265285.

4. Moont, G.; Gabb, H. A.; Sternberg, M. J., Proteins 1999, 35, (3), 364-373.

5. Rehr, J. J. M. d. 1., J.; Zabinsky, S. I.; Albers, R. C., J. Am. Chem. Soc. 1991, 113, 5135-5138.

6. Stern, E. A.; Newville, M.; Ravel, B.; Haskel, D.; Yacoby, Y., Physica B 1995, $208 \& 209,117-122$.

7. Zabinsky, S. I.; Rehr, J. J.; Ankudinov, A.; Albers, R. C.; Eller, M. J., Physical Review. B. Condensed Matter. 1995, 52, (4), 2995-3009.

\section{References in the main text that contain 16 authors or more:}

3. Folgueras, A. R.; Pendas, A. M.; Sanchez, L. M.; Lopez-Otin, C.; Pendas, A. M.; Folgueras, A. R.; Llano, E.; Caterina, J.; Frerard, F.; Rodriguez, F.; Astudillo, A.; Noel, A.; Birkedal-Hansen, H.; Lopez-Otin, C.; Santos-Juanes, J.; Bernaldo de Quiros, J. F.; Galache Osuna, C.; Sanchez del Rio, J.; Allende, M. T.; Folgueras, M. V.; Soto de Delas, J., Matrix metalloproteinases in cancer: from new functions to improved inhibition strategies. Diet-induced obesity and reduced skin cancer susceptibility in matrix metalloproteinase 19-deficient mice. Apocrine carcinoma, adenopathies, and raised TAG72 serum tumor marker. Int J Dev Biol 2004, 48, (5-6), 411-424.

37. Case, D. A.; Pearlman, D. A.; Caldwell, J. W.; Cheatham III, T. E.; Wang, J.; Ross, W. S.; Simmerling, C. L.; Darden, T. A.; Merz, K. M.; Stanton, R. V.; Cheng, A. L.; Vincent, J. J.; Crowley, M.; Tsui, V.; Gohlke, H.; Radmer, R. J.; Duan, Y.; Pitera, J.; Massova, I.; Seibel, G. L.; Singh, U. C.; Weiner, P. K.; A, K. P., AMBER 7. ed.; University of California: San Francisco, 2002; 'Vol.' p. 